\title{
Endogenous Opsin 3 (OPN3) Protein Expression in the Adult Brain Using a Novel OPN3-mCherry Knock-In Mouse Model
}

\author{
[DLauren E. Olinski, ${ }^{1}{ }^{\circledR}$ Ayumi C. Tsuda $^{2}{ }^{2}$ Julie A. Kauer, ${ }^{3}$ and Elena Oancea ${ }^{2}$
}

https://doi.org/10.1523/ENEURO.0107-20.2020

${ }^{1}$ Department of Molecular Biology, Cell Biology, and Biochemistry, Brown University, Providence, RI 02912 ,

${ }^{2}$ Department of Molecular Pharmacology and Physiology, Brown University, Providence, RI 02912, and ${ }^{3}$ Department of Psychiatry, Stanford University, Stanford, CA 94305

\section{Visual Abstract}

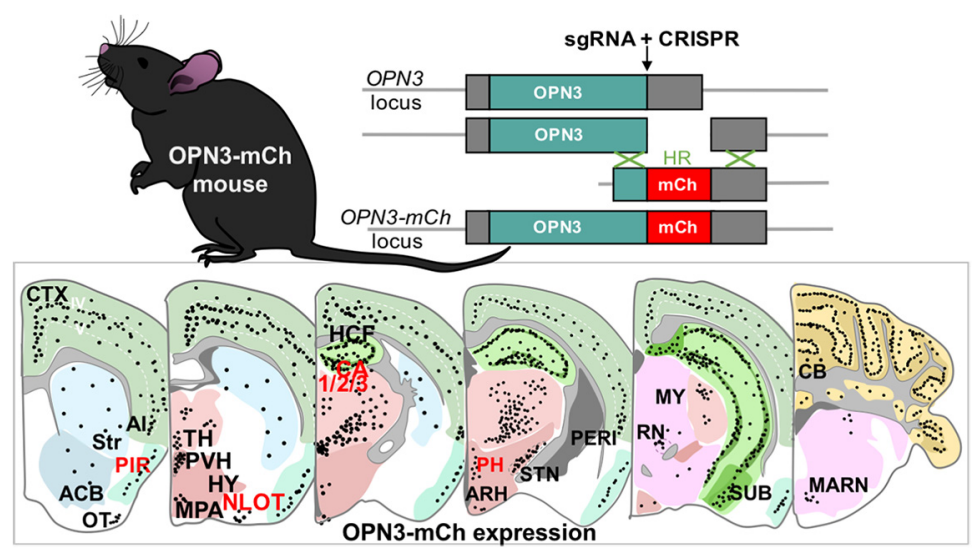

The opsins have been studied extensively for their functions in visual phototransduction; however, the mechanisms underlying extraocular opsin signaling remain poorly understood. The first mammalian extraocular opsin to be discovered, opsin 3 (OPN3), was found in the brain more than two decades ago, yet its function remains unknown. A significant hindrance to studying OPN3 has been a lack of specific antibodies against mammalian OPN3, resulting in an incomplete understanding of its expression in the brain. Although Opn3 promoter-driven reporter mice have been generated to examine general OPN3 localization, they lack the regulated expression of the endogenous protein and the ability to study its subcellular localization. To circumvent these issues, we have created a knock-in OPN3-mCherry mouse model in which the fusion protein OPN3-mCherry is expressed

\section{Significance Statement}

Before the current report, there had been a significant lack of encephalic opsin 3 (OPN3) protein characterization, likely driven by the absence of murine OPN3 antibodies. This study describes a novel OPN3-mCherry knock-in mouse that we used to analyze endogenous OPN3 protein expression across the brain. We have uncovered new aspects of OPN3: localization to previously undocumented brain subregions, expression in GABAergic neurons and non-neuronal cells, and punctate subcellular localization in the soma. Our OPN3 expression map is an invaluable step toward discovering its elusive encephalic functions. The OPN3-mCherry mouse will facilitate investigation of OPN3 not only in the brain, but also across the entire organism, a useful feature as OPN3 is emerging as a possible mediator of phototransduction outside the brain. 
under the endogenous Opn3 promoter. Viable and fertile homozygotes for the OPN3-mCherry allele were used to create an extensive map of OPN3-mCherry expression across the adult mouse brain. OPN3-mCherry was readily visualized in distinct layers of the cerebral cortex (CTX), the hippocampal formation (HCF), distinct nuclei of the thalamus, as well as many other regions in both neuronal and non-neuronal cells. Our mouse model offers a new platform to investigate the function of OPN3 in the brain.

Key words: CRISPR/Cas9; encephalopsin; mouse; OPN3; opsin

\section{Introduction}

Opsins form a family of light-sensitive G-protein-coupled receptors (GPCRs) that function in a multitude of phototransduction mechanisms (Terakita, 2005). We depend on light to see, and as such, opsins have consistently been the research focus behind visual phototransduction, with retina-residing opsins being the most frequently investigated. Only recently have mammalian extraretinal opsins and their non-image forming functions become a major field of research.

The first opsin to be identified as having expression outside of the eye was opsin 3 (OPN3; Blackshaw and Snyder, 1999). OPN3 was initially named encephalopsin for its abundant expression in the mouse brain (Blackshaw and Snyder, 1999); it was later termed panopsin after discovery of its widespread expression in peripheral organs in mammals (Halford et al., 2001). Despite its discovery more than two decades ago, OPN3 remains one of the least characterized opsins and its expression profile and function are unknown in many of the tissues where it is expressed. The studies published thus far have focused primarily on OPN3 expression or function in the skin (for review, see Olinski et al., 2020), lungs (White et al., 2008; Barreto Ortiz et al., 2018; Yim et al., 2019), colon (Yoshimoto et al., 2018), liver (Jiao et al., 2012), and adipocytes (Nayak et al., 2020; Sato et al., 2020), but OPN3 has yet to gain attention in the brain, where it was first discovered.

Received March 19, 2020; accepted June 4, 2020; First published July 31, 2020.

The authors declare no competing financial interests.

Author contributions: L.E.O., A.C.T., and E.O. designed research; L.E.O. and A.C.T. performed research; L.E.O., J.A.K., and E.O. analyzed data; L.E.O., J.A.K., and E.O. wrote the paper.

This work was supported by The National Institute of General Medical Sciences Grant T32 GM077995 (to L.E.O.), Brown University Carney Graduate Award in Brain Science (to L.E.O.), the National Institute of Arthritis and Musculoskeletal and Skin Diseases Grant R01 AR066318 (to E.O.), and the Brown University SEED Award GR300157 (to E.O.). The Brown University Mouse Transgenic and Gene Targeting Facility was supported by a grant from the National Institute of General Medical Sciences (P30 GM103410) from the National Institutes of Health.

Acknowledgements: We thank members of the Oancea laboratory for their feedback, especially $H$. Haddad and Dr. R. Ozdeslik for their discussions about OPN3. We also thank M. Leyrer and Dr. D. Berson for generously providing the ChAT antibody and Dr. B. Pradier and O. Baytas at Brown University for their assistance in refining staining protocols and sectioning techniques. We thank D. Savas and J. Trotman for their help with mouse husbandry and care.

Correspondence should be addressed to Elena Oancea at elena oancea@brown.edu.

https://doi.org/10.1523/ENEURO.0107-20.2020

Copyright (C 2020 Olinski et al.

This is an open-access article distributed under the terms of the Creative Commons Attribution 4.0 International license, which permits unrestricted use, distribution and reproduction in any medium provided that the original work is properly attributed.
Initially, OPN3 mRNA was identified by in situ hybridization in several murine brain areas including: cerebral cortex (CTX), striatum, preoptic areas, lateral thalamus, paraventricular nucleus of the hypothalamus (PVH), spinal cord, and cerebellar Purkinje cells (Blackshaw and Snyder, 1999). It was only in 2012 that OPN3 protein expression was examined in the adult mouse brain through immunofluorescence and immunoblotting for endogenous OPN3 (Nissilä et al., 2012). However, this study was unable to specifically dissect OPN3 expression across the entire brain, and some results were in direct opposition to previous reports; for example, OPN3 mRNA was highly expressed in the testes (Blackshaw and Snyder, 1999; Halford et al., 2001), but no expression was found there by Nissilä et al. (2012). The promiscuity of many OPN3 antibodies may have been the cause of this discrepancy. Years later, a report in 2018 characterized OPN3 protein expression in giant choline acetyltransferase (ChAT)-expressing and small non-ChATexpressing neurons of the monkey striatum (El Massri et al., 2018), but this study was again limited by the specificity of commercially available OPN3 antibodies.

To circumvent the dependence on antibodies for the complete characterization of OPN3 across tissues, we have used CRISPR/Cas9 gene editing to create a knockin mouse model which expresses mCherry $(\mathrm{mCh})$-tagged OPN3 under the endogenous Opn3 promoter. The advantage of this reporter mouse over other models is that OPN3-mCh fusion protein is expressed under the native promoter, preserving endogenous levels of OPN3 with native expression across development. This is fundamental for furthering OPN3 characterization as OPN3 has been shown to be developmentally regulated, at least in the brain (Blackshaw and Snyder, 1999; Lein et al., 2007).

Our novel mouse model allows unequivocal analysis of OPN3 protein expression by monitoring $\mathrm{mCh}$ fluorescence in an entire mouse. Consequently, our first endeavor with this mouse model was to enable the complete characterization of endogenous OPN3 expression in the adult brain. Animals homozygous for the knock-in mCh-tagged Opn3 allele were used to systematically characterize OPN3-mCh expression in the adult mouse brain from coronal, sagittal, and horizontal sections. We examined OPN3-mCh-expressing cells for coexpression of neuronal, astrocytic, ChAT, and GABAergic markers to gain further insight into the types of cells expressing OPN3 for future functional studies.

\section{Materials and Methods}

\section{Generation of the OPN3-mCh knock-in mouse}

In conjunction with the Brown University Mouse Transgenic and Gene Targeting Facility, CRISPR/Cas9 gene editing technology was used to insert mCherry 
A sgRNA1, sgRNA2, sgRNA3

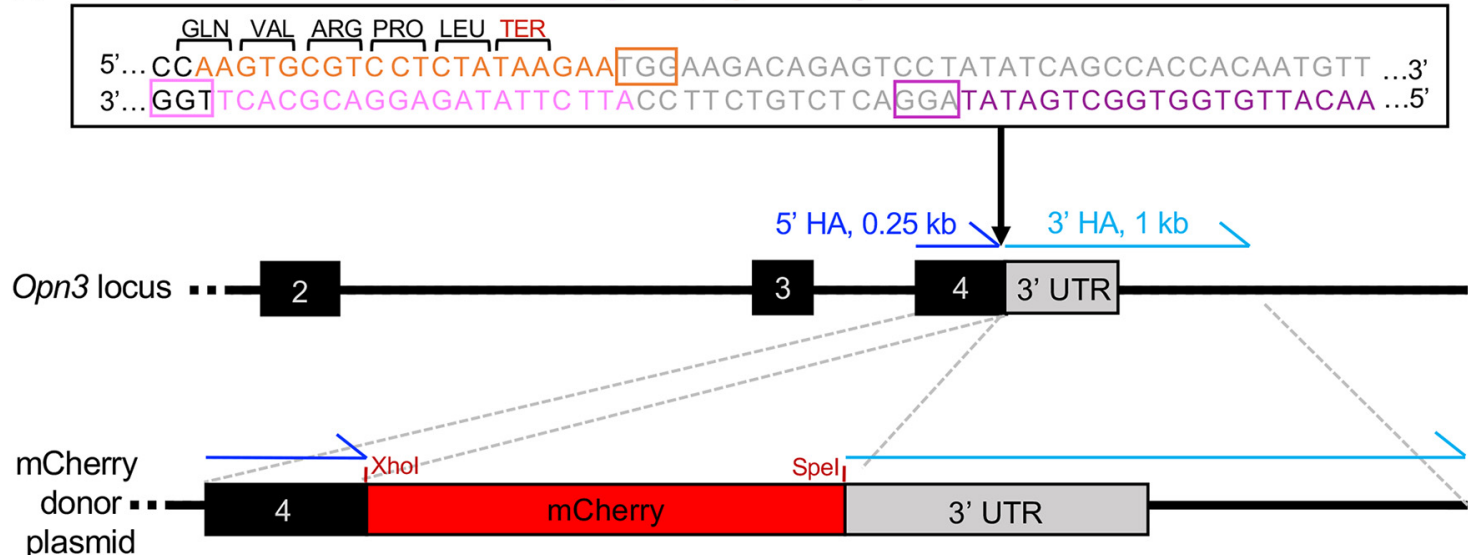

plasmid HR
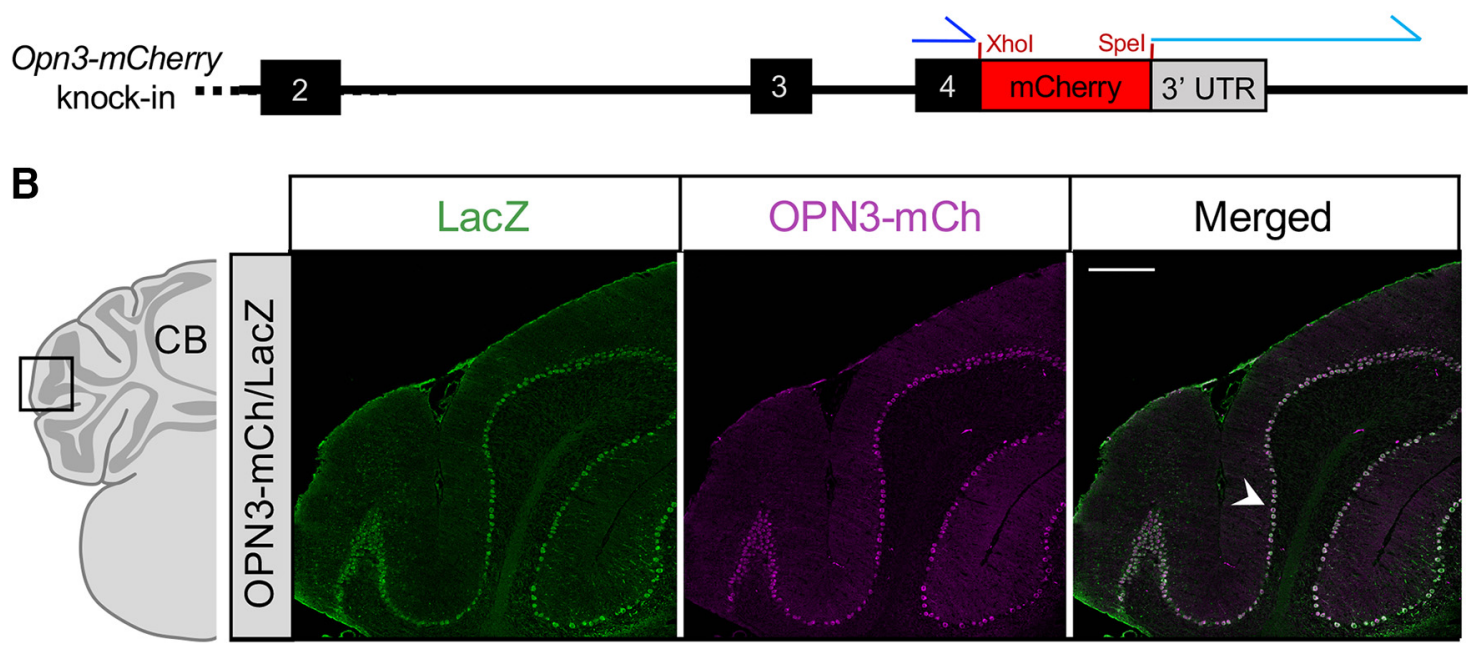

Figure 1. OPN3-mCh knock-in mouse model generation using CRISPR/Cas9 editing. $\boldsymbol{A}$, Targeting strategy for the OPN3-mCh knock-in allele. mCherry ( $\mathrm{mCh}$ ) was inserted in-frame immediately before the termination codon in exon 4 of mouse Opn3. The resulting fusion protein expresses mCh fused at the $\mathrm{C}$ terminus of OPN3 via a short linker. Double-stranded DNA sequence highlights sgRNA1 (pink), sgRNA2 (orange), sgRNA3 (purple), Opn3 exon 4 (black), and Opn3 3' UTR (gray) with colored boxed areas indicating PAM sites. HR: homologous recombination, HA: homology arm, TER: termination codon. Primers for genotyping are shown in Extended Data Figure 1-1. B, Validation of OPN3-mCh brain expression using the OPN3-mCh/LacZ mouse. The OPN3-mCh/LacZ mouse was generated by crossing our OPN3-mCh knock-in mouse with a mouse in which OPN3 was replaced by LacZ

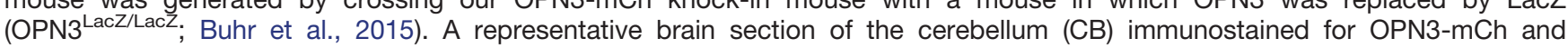
LacZ shows significant overlap in Purkinje cells (arrowhead). Scale bar: $250 \mu \mathrm{m}$.

(mCh) in-frame at the carboxy (C) terminus of the endogenous Opn3 locus, before the termination codon. The resultant knock-in mouse model expresses the fusion protein OPN3-mCh under the control of the endogenous Opn3 promoter. The Transgenic Facility microinjected $>200$ C57BL/6 embryos with $10 \mathrm{ng} / \mu \mathrm{l}$ each of (1) sgRNA1/sgRNA2 or sgRNA1/sgRNA3 (Table 2: Primers and sgRNA sequences; made in-house through in vitro transcription with MEGAshortscript T7 Transcription kit, Ambion), which target nucleotides immediately surrounding the Opn3 termination codon; (2) Cas9 mRNA (Alt-R S.p. Cas9 Nuclease V3, IDT); and (3) a donor plasmid comprised of a pUC57 backbone (Addgene) with a $0.25 \mathrm{~kb} 5^{\prime}$ homology arm followed by a linker (LEGAGA), $\mathrm{mCh}$, then a $1.0 \mathrm{~kb} 3^{\prime}$ homology arm (Fig. 1A). Embryos were implanted in the oviducts of pseudopregnant Swiss
Webster females (Taconic Biosciences). Two $\mathrm{G}_{0}$ founders (one from sgRNA1/sgRNA2 and one from sgRNA1/ sgRNA3) were confirmed to have $\mathrm{mCh}$ inserted in-frame by long-range and short-range PCR. $\mathrm{G}_{0}$ founders were crossed with $\mathrm{C} 57 \mathrm{BL} / 6$ mice to obtain a stock of $\mathrm{G}_{1}$ OPN3-mCh/+ heterozygotes. After backcrossing, heterozygous OPN3-mCh/+ mice were interbred to obtain homozygous OPN3-mCh/OPN3-mCh mice (herein referred to as OPN3-mCh mice) which were confirmed by short-range PCR. Neither the heterozygous nor homozygous OPN3-mCh mice have any observable phenotype. We did not observe any differences in OPN3-mCh localization between the heterozygote and the homozygote, only differences in $\mathrm{mCh}$ fluorescence levels, as expected. Thus, we performed all staining experiments (unless otherwise stated) using sections from homozygous 
Table 1: Antibodies

\begin{tabular}{lll}
\hline Antibody & Company & Concentration \\
\hline Anti-mCherry & KeraFAST (EMU105) & $1: 500$ \\
Anti- $\beta$-galactosidase (LacZ) & GeneTex (GTX77365) & $1: 500$ \\
Anti-MAP2 & Santa Cruz Biotechnology (sc-74421) & $1: 200$ \\
Anti-GFAP & Santa Cruz Biotechnology (sc-33673) & $1: 200$ \\
Anti-GAD67 & Millipore Sigma (MAB5406) & $1: 500$ \\
Anti-ChAT & Millipore Sigma (AB144P) & $1: 500$ \\
Goat anti-mouse lgG2a-568 & Life Technologies (A21134) \\
Donkey anti-mouse-647 & Invitrogen (A31571) & $1: 500$ \\
Donkey anti-rabbit-488 & Invitrogen (A32814) & $1: 500$ \\
Donkey anti-rabbit-594 & Invitrogen (A32754) & $1: 500$ \\
Donkey anti-chicken-647 & Jackson ImmunoResearch (703-605-155) & $1: 500$ \\
\hline
\end{tabular}

mice because the fluorescence intensity was greater. OPN3-mCh male and female mice are fertile, but as with any genetically-modified animal, breeding efficiency in the OPN3-mCh mice is less robust than in wild-type (WT) C57BL/6 mice. The OPN3-mCh female mice tend to have smaller litters (approximately two to four pups), and we have observed them to be more prone to cannibalizing their young.

\section{Origin of other mouse lines used}

C57BL/6 mice originated from The Jackson Laboratory. The OPN3 knock-out (OPN3 ${ }^{\text {LacZ/LacZ) }}$ transgenic mice (Buhr et al., 2015) were a generous gift from K.-W. Yau at Johns Hopkins University. In brief, this line was generated by Buhr et al. (2015) by crossing OPN3 ${ }^{\text {flox/flox }}$ mice with Sox2-Cre mice. OPN3 ${ }^{\text {flox/flox }}$ mice contained a targeting construct for inserting a flippase recognition targetflanked LacZ cassette upstream of Opn3 exon 2. Cre-recombination led to global deletion of endogenous Opn3 and insertion of LacZ under the Opn3 promoter and thus creation of the OPN $3^{\text {LacZ/LacZ }}$ line.

\section{Animals}

All animal care and procedures in this study were performed in accordance with the Brown University animal care committee's regulations. Animals were housed socially with ad libitum access to chow and water in a lightcontrolled (12/12 h light/dark cycle) and temperature-controlled $\left(21.5-23.5^{\circ} \mathrm{C}\right)$ facility. Both male and female mice were used, as there was no significant difference in OPN3 expression between sexes. Genotyping primers for OPN3-mCh and OPN3 ${ }^{\text {LacZ/LacZ }}$ are in Table 2: Primers and sgRNA sequences; and Extended Data Figure 1-1.

\section{Tissue collection}

Ten mice (4-12 months old; five OPN3-mCh, three WT, two OPN3-mCh/LacZ) were deeply anesthetized with $100 \mathrm{mg} / \mathrm{kg}$ ketamine and $0.25 \mathrm{mg} / \mathrm{kg}$ dexmedetomidine via subcutaneous injection and transcardially perfused with 50 $\mathrm{ml}$ of ice-cold PBS, followed by $50 \mathrm{ml}$ of freshly prepared, ice-cold 4\% paraformaldehyde. Once brains were removed, they were postfixed in $4 \%$ paraformaldehyde for $<18 \mathrm{~h}$ at $4^{\circ} \mathrm{C}$. After rinsing thoroughly with cold PBS, brains were cryoprotected in increasing concentrations of
sucrose-PBS solutions (10\%, 20\%, 30\%) over 3 d (or until the brain sank to the bottom of the holding container). Brains were flash-frozen for $10-15 \mathrm{~s}$ in a slurry of 2methylbutane and dry ice and stored at $-80^{\circ} \mathrm{C}$ until sectioning on a cryostat (Leica CM3050S) at 25-30 $\mu \mathrm{m}$. Sections were stored in an antifreeze solution [20\% (v/v) glycerol, $20 \%(\mathrm{v} / \mathrm{v})$ ethylene glycol in $1 \times \mathrm{PBS}]$ at $-20^{\circ} \mathrm{C}$ until immunostaining.

\section{Free-floating immunofluorescence}

All antibodies and concentrations can be found in Table 1: Antibodies Brain sections were washed with PBS $2 \times 5$ min then permeabilized with $0.3 \%$ PBS-Triton X-100 (PBST) $3 \times 10 \mathrm{~min}$. Brains were blocked with a solution of $5 \%$ donkey serum and 1\% BSA in PBS for $\sim 2 \mathrm{~h}$ at room temperature $(\mathrm{RT})$. Sections were incubated in primary antibody diluted in the above blocking solution for 24$72 \mathrm{~h}$ at $4^{\circ} \mathrm{C}$ with light shaking (50-70 rpm). Sections were then washed in PBS $3 \times 10 \mathrm{~min}$ before blocking/permeabilizing in a solution of $5 \%$ donkey serum in $0.3 \%$ PBST for $\sim 2 \mathrm{~h}$ at RT (this step was omitted for MAP2, GAD67, and GFAP immunostainings). Sections were incubated in secondary antibody diluted in $0.3 \%$ PBST for $\sim 2 \mathrm{~h}$ at RT with

\section{Table 2: Primers and sgRNA sequences}

\begin{tabular}{ll}
\hline Sequence & Use \\
\hline ATTCTTATAGAGGACGCACT & sgRNA1 \\
AAGTGCGTCCTCTATAAGAA & sgRNA2 \\
AACATTGTGGTGGCTGATAT & sgRNA3 \\
CTCCGTTGTTTCTCTCTGCAG & Primer 360 Full length \\
& PCR-Forward \\
ACGCCAGATGCTCATCTTG & Primer 362 OPN3 WT- \\
& Reverse \\
CTCAGTGACTTCCAACTCAAGG & Primer 367 Full length \\
& PCR-Reverse \\
CTCTACTCGAGGGAGCAGGAG & Primer 369 3' Long Range \\
& PCR-Forward \\
AAGCGCATGAACTCCTTGATG & Primer 371 OPN3-mCh- \\
& Reverse \\
CTTGTACAGCTCGTCCATGC & Primer 372 5' Long Range \\
& PCR-Reverse \\
TTATGGCCCACACCAGTGGC & Primer OPN3 KO-Forward \\
TGTACCGTGGACTGGAGATC & Primer OPN3 WT-Forward \\
CAAG & Primer OPN3-Reverse \\
GTTCCCACACACGACCTGCTC &
\end{tabular}


light shaking. After a final wash in PBS $3 \times 10$ min and $a<1$ min rinse in deionized water, the sections were placed on SuperFrost Plus slides (Thermo Fisher Scientific) and mounted with VECTASHIELD Antifade Mounting Media with DAPI (Vector Laboratories). For Nissl stains, free-floating sections were rinsed $3 \times 5$ min in PBS before staining with NeuroTrace 530/615 Red Fluorescent Nissl Stain (Thermo Fisher Scientific) according to manufacturer's instructions (omitting initial rehydration step). Sections were mounted as noted above.

\section{Microscopy, image acquisition, and analysis}

Fluorescence staining was viewed using either an Olympus FV3000 Confocal Laser Scanning Microscope, Zeiss LSM 800 Confocal Laser Scanning Microscope or Nikon Ti2-E Fluorescence Microscope. The Olympus FV3000 has an inverted configuration and is equipped with a resonant scanner and four detectors, two standard Metal Alkalide and two high-sensitivity GaAsP. The Zeiss LSM 800 is based on an Axio Imager Z2 microscope and is equipped with a three high-sensitivity GaAsP detectors. Widefield images in Figure 2 were acquired on the Nikon Ti2-E microscope using a $10 \times$ objective and stitched using Nikon-based NIS-Elements software. Zoomed regions were acquired on the Olympus FV3000 or Zeiss LSM 800 microscopes using 10-30 $\times$ objectives and stitched using cellSens (Olympus) or Zen Blue (Zeiss) software. The images from OPN3-mCh mice and WT control mice were collected using identical acquisition settings. FIJI/ImageJ $(\mathrm{NIH})$ was used to adjust image brightness/contrast, using the same parameters for images obtained from OPN3$\mathrm{mCh}$ and WT control mice. Scale bars were added to final images using FIJI/ImageJ. The density of OPN3-mCh-expressing cells by area in Figure 3 was determined based on serial coronal, sagittal, and horizontal sections from four OPN3-mCh mice. The number of positive OPN3-mCh cells was determined using DAPI counterstain via visual analysis. Brain regions were named using conventions from Franklin and Paxinos (Paxinos and Franklin, 2013). Anatomy outlines, and color schemes in figures are based on the Allen Mouse Brain Atlas (Lein et al., 2007). Estimates of the abundance of OPN3-mCh-expressing cells in Figure 4 were determined from both widefield and confocal images in coronal, horizontal, and sagittal planes. Image contrast was adjusted and a merged image of the DAPI channel on the $\mathrm{mCh}$ channel was used. Abundance was determined through visual analysis of $\mathrm{mCh}$ positive cells over total cell number per brain region per section. The relative abundance of OPN3mCh cells was noted as very high: ++++ ; high: +++ ; moderate: ++; or low: +, corresponding approximately to the following percentages of OPN3-mCh+ cells/total cells: very high: $>70 \%$; high: $40-70 \%$; moderate: $10-40 \%$; low: 5-10\%.

\section{Results}

\section{Generation of the OPN3-mCh mouse}

The OPN3-mCh knock-in mouse was generated using CRISPR/Cas9 technology for efficient and precise insertion of the OPN3-mCh fusion protein under the endogenous Opn3 promoter. A combination of two sgRNAs targeting the junction between the last exon of mouse Opn3 (exon 4) and the 3' untranslated region (UTR) were injected along with Cas9 mRNA and a donor plasmid containing mCh into C57BL/6 embryos (Fig. 1A). A more extensive description of procedures can be found in Materials and Methods. The fluorescent tag $\mathrm{mCh}$ was chosen over other red fluorophores (such as tdTomato) based on availability of reliable antibodies against $\mathrm{mCh}$, in the event that fluorescent expression on its own was too dim to detect. We used the same OPN3 fusion protein construction (mCh attached to the $\mathrm{C}$ terminus of OPN3 via a flexible linker) that was previously demonstrated not to interfere with localization or function of the OPN3 protein in melanocytes (Ozdeslik et al., 2019). After confirming successful knock-in of $\mathrm{mCh}$ at the direct $\mathrm{C}$ terminus of Opn3, heterozygous mice were backcrossed and bred to create mice homozygous for OPN3-mCh. Homozygous OPN3$\mathrm{mCh}$ mice were used for experiments unless stated.

The endogenous $\mathrm{mCh}$ fluorescence in brain sections from OPN3-mCh mice was too weak to detect. We therefore used a validated anti-mCh antibody to amplify the endogenous $\mathrm{mCh}$ signal as a proxy for OPN3-mCh expression. The same anti-mCh antibody was used for immunostaining skin sections from OPN3-mCh mice and showed a detectable and reliable signal in cells known to express OPN3, but not in control mice without OPN3- mCh (Olinski et al., 2020). We next sought to confirm the fidelity of OPN3-mCh expression to endogenous OPN3 expression in the brain. In previous publications we used an OPN3 antibody to detect endogenous OPN3 (Ozdeslik et al., 2019), but the discontinuation of this antibody and lack of a suitable replacement led us to pursue an alternative approach to confirm that OPN3-mCh in the knock-in mouse can successfully be detected in areas where endogenous OPN3 is known to be expressed. We crossed the previously generated OPN3 $\beta$ galactosidase (LacZ)-reporter mouse (OPN3 ${ }^{\text {LacZ/LacZ; Buhr et al., 2015) }}$ with our OPN3-mCh knock-in mouse to create OPN3-mCh/ LacZ mice. These mice, under the Opn3 promoter, expressed one LacZ allele and one OPN3-mCh allele. Figure $1 B$ is a representative image of the cerebellum of the OPN3$\mathrm{mCh} / \mathrm{LacZ}$ mouse coimmunostained for LacZ and $\mathrm{mCh}$ showing LacZ and $\mathrm{mCh}$ signal overlap and specificity to Purkinje cells of the cerebellum. This indicates that the OPN3-mCh expression in the OPN3-mCh mouse line is localized consistently with the Opn3 promoter-controlled LacZ reporter, as expected. Because this was a heterozygous mouse for the OPN3-mCh allele, the fluorescence expression was lower than for the homozygous mice and thus was best in brain areas with high OPN3 expression, namely, the cerebellum. We also used the cerebellum to test specificity because this region is undisputed as an area with high OPN3 expression in the brain (Blackshaw and Snyder, 1999; Lein et al., 2007; Nissilä et al., 2012). For a more complete characterization of OPN3 expression in the brain, we used homozygous OPN3-mCh mice to identify and estimate the expression level of OPN3 in different brain areas. To ensure the specificity of the mCh antibody, we immunostained brain sections from OPN3-mCh and WT mice. We saw 

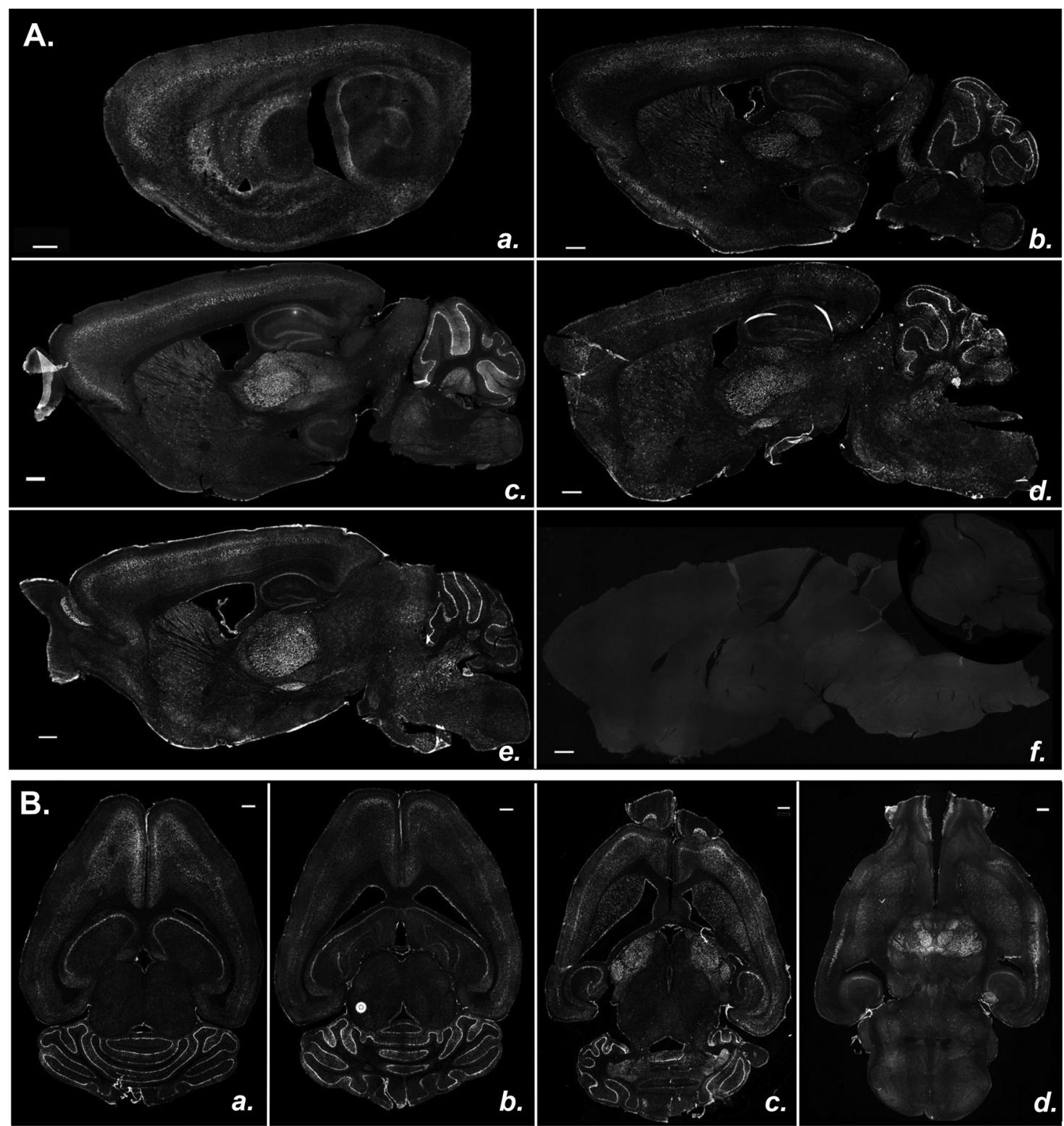

Figure 2. Endogenous OPN3-mCh expression across the adult OPN3-mCh mouse brain. $\boldsymbol{A a}-\boldsymbol{A} \boldsymbol{e}$, Fluorescent images of sagittal brain sections from adult OPN3-mCh mice, immunostained with anti-mCh antibody and arranged in a lateral-to-medial manner. Note high OPN3-mCh expression in the thalamus, cerebellum, HCF, and CTX. Af, Representative fluorescent image of a sagittal brain section from a WT mouse immunostained with anti-mCh antibody, imaged under the same settings as the sections in $\boldsymbol{A a - A \boldsymbol { e }}$; it has negligible fluorescence signal, as expected. Scale bars: $500 \mu \mathrm{m}$. Ba-Bd, Fluorescent images of horizontal brain sections from adult OPN3-mCh mice immunostained with anti-mCh antibody and arranged in a dorsal-to-rostral manner. Bright circle in left midbrain of $\boldsymbol{B} \boldsymbol{b}$ is an imaging artifact. Scale bars: $500 \mu \mathrm{m}$.

specific and consistent expression of $\mathrm{mCh}$ across serial sections from OPN3-mCh mice and no mCh immunoreactivity in WT sections (Fig. 2). OPN3-mCh sections in the following figures were stained with anti-mCh antibody to amplify OPN3-mCh signal and matching WT brain slices were used as controls for all experiments.

\section{Endogenous OPN3-mCh is present in several brain regions in neuronal and non-neuronal cell types}

Sagittal and horizontal OPN3-mCh brain sections revealed unique patterns of expression (Fig. 2). We obtained sagittal (Fig. 2A), horizontal (Fig. 2B), and coronal (Fig. 3; Extended Data Fig. 3-1) sections from OPN3-mCh mouse brains to perform semi-quantitative analysis of OPN3$\mathrm{mCh}$ expression in individual regions. Figure 3 shows coronal depictions of OPN3-mCh expression profiles in which the density of the black dots (each representing $\sim 10$ OPN3-mCh cells) shows distinct patterns of known expression across regions. Areas of new OPN3 expression identified using our OPN3-mCh mouse are noted in red (Fig. 3). Representative fluorescent images can be found in Extended Data Figure 3-1. OPN3-mCh exhibited a double-banded pattern in the CTX, most prominently 

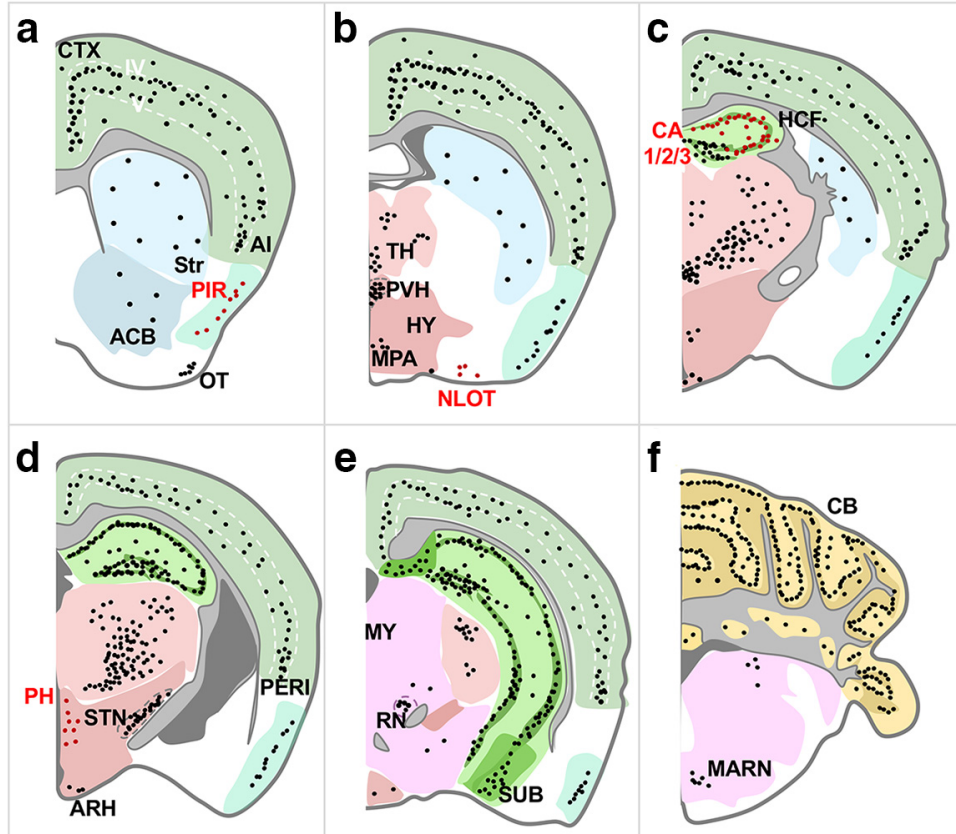

Figure 3. Semi-quantitative analysis of OPN3 distribution and abundance in the adult OPN3-mCh mouse brain. a-f, Line drawings of coronal sections arranged in a rostral-to-caudal manner with each black dot representing $\sim 10$ OPN3-mCh-positive cells, averaged from coronal, sagittal, and horizontal sections from four OPN3-mCh mice immunostained and imaged as in Figure 2. Red labels and dots indicate new regions of OPN3 expression revealed by our OPN3-mCh mouse. Different colors correspond to different brain regions as labeled; dark gray corresponds to ventricles and light gray to fiber tracts. As indicated by the density of black dots, high expression of OPN3-mCh was detected in Layers IV-V of the CTX, HCF, ventral and lateral nuclei of the thalamus (TH), and cerebellum (CB). Representative fluorescent images of coronal sections used for analysis are shown in Extended Data Figure 3-1. ACB: nucleus accumbens, Al: agranular insular area, ARH: arcuate nucleus, HY: hypothalamus, MARN: magnocellular reticular nucleus, MY: medulla, NLOT: nucleus of the lateral olfactory tract, OT: olfactory tubercle, PERI: perirhinal area, PH: posterior hypothalamic nucleus, PIR: piriform area, RN: red nucleus, STN: subthalamic nucleus, Str: striatum, SUB: subiculum.

within Layers IV and V; sparse, but even distribution within the striatum; ventral-specific and lateral-specific expression in the thalamus; and robust expression in the Purkinje cell layer of the cerebellum. These observations are summarized according to the $\mathrm{mCh}$ immunostaining intensity of each region in Figure 4.

Our first objective was to broadly determine in which cell types OPN3-mCh is expressed. From other studies, it was known that OPN3 is expressed in at least some neurons, like the Purkinje cells of the cerebellum. It was unknown, however, whether OPN3 was also present in non-neuronal cells within the brain. As we conducted our analysis of the OPN3-mCh brain, it soon became evident that OPN3 is expressed in non-neuronal cell types, notably in the hippocampal formation (HCF). To determine neuronal expression of OPN3-mCh, we coimmunostained sections with $\mathrm{mCh}$ and the neuronal marker microtubule-associated protein 2 (MAP2; Cassimeris and Spittle, 2001). Figure 5 shows representative examples of three brain regions with neuronal overlap: the CTX, cerebellum, and HCF. Within the CTX, OPN3-mCh was present in large, pyramidal neurons in the somatomotor area, Layer V (Fig. 5Aa). In the cerebellum, there was strong neuronal expression of OPN3-mCh in the Purkinje cell layer, molecular layer, and to a lesser extent, the granule layer (Fig. 5Ac). Expression in the HCF was less homogenous with regard to cell type. OPN3-mCh staining was apparent in the pyramidal cell layers in Ammon's horn (CA) CA1/2/3 and the granule layer and hilus of the dentate gyrus (DG; Fig. $5 A b$ ) as well as small cells with multiple processes that were not clearly labeled by MAP2 (Fig. 5Ab). These are likely astrocytes, as they express glial fibrillary acidic protein (GFAP; Messing and Brenner, 2003; Fig. 5B); OPN3-mCh colocalized with GFAP in most of the GFAP+ astrocytes. This is the first instance of OPN3 identification in a non- neuronal cell type.

We also noticed that many of the regions in which OPN3 is expressed (Figs. 2-4) are known to contain populations of GABAergic cells (Tamamaki et al., 2003): the CTX, cerebellum, and striatum, among other regions. To further dissect whether OPN3-mCh is found preferentially in GABAergic cells, we stained OPN3-mCh sections for glutamic acid decarboxylase 67 (GAD67), one of two enzymes that catalyze the conversion of glutamate to GABA (Pinal and Tobin, 1998; Fig. 6). GAD67 was concentrated primarily in the soma as previously described (Asada et al., 1997; Kanaani et al., 2010), similar to OPN3-mCh expression. A widefield view of the coimmunostaining indicated that some OPN3-mCh+ cells were also GAD67+, such as cells in the Purkinje layer of the cerebellum. However, not all GAD67+ cells were OPN3-mCh+, as 

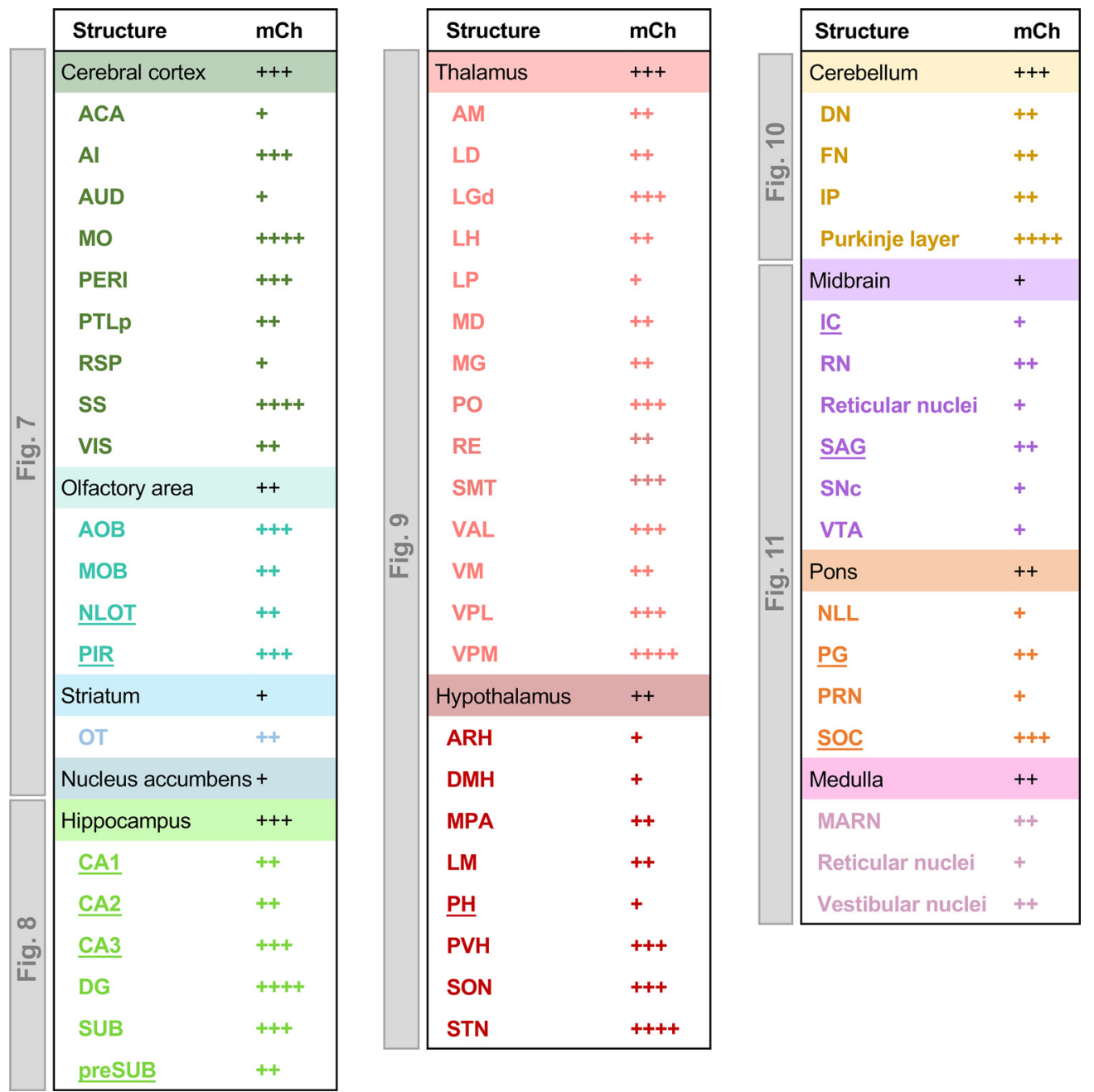

Figure 4. R>elative abundance of OPN3-mCh immunoreactivity in different regions of the OPN3-mCh mouse brain. Estimated abundance of OPN3-mCh-expressing cells in each brain subregion was based on the fraction of mCh immunoreactive cells over total cells visualized by DAPI in coronal, sagittal, and horizontal sections across the adult OPN3-mCh mouse brain. ++++ : highest density, +++ : high density, ++ : moderate density, + : low density. Newly identified brain areas expressing OPN3-mCh are underlined. Similarities and differences of OPN3 expression between our identified brain regions and previous reports are in Extended Data Figure 4-1. ACA: anterior cingulate area, Al: agranular insular area, AM: anteromedial nucleus, ARH: arcuate nucleus, AUD: auditory area, DMH: dorsomedial nucleus, DN: dentate nucleus, FN: fastigial nucleus, IC: inferior colliculus, IP: interposed nucleus, LGd: dorsal lateral geniculate nucleus, LH: lateral habenula, LM: lateral mammillary nucleus, MARN: magnocellular reticular nucleus, MD: mediodorsal nucleus, MG: medial geniculate complex, MO: somatomotor area, MOB: main olfactory bulb, NLL: nucleus of the lateral lemniscus, NLOT: nucleus of the lateral olfactory tract, OT: olfactory tubercle, PG: pontine grey, PERI: perirhinal area, PH: posterior hypothalamic nucleus, PIR: piriform area, preSUB: presubiculum, PRN: pontine reticular nucleus, PTLp: posterior parietal association area, RE: nucleus of reuniens, RN: red nucleus, RSP: retrospenial area, SAG: nucleus sagulum, SNc: substantia nigra compacta, SON: supraoptic nucleus, SOC: superior olivary complex, SS: somatosensory area, STN: subthalamic nucleus, SUB: subiculum, VIS: visual area, VTA: ventral tegmental area.

evidenced by the GABAergic reticular nucleus of the thalamus, which lacks OPN3-mCh expression (Fig. 6A). At greater magnification in the CTX, Layers IV and V showed strong signal overlap between GAD67 and OPN3-mCh. OPN3-mCh was also present to a lesser extent in Layers II-III and VI, which have less GAD67 expression (Fig. 6Ba). In the HCF, GAD67 staining revealed that OPN3-mCh was present in GAD67+ cells around the pyramidal and granule layers. Within the DG, OPN3mCh was expressed in GAD67+ cells, but also in cells devoid of GAD67, putatively glutamatergic mossy cells in the hilus (Fig. 6Bb). As expected from previous studies, OPN3-mCh was also present in GABAergic Purkinje cells and in many GABAergic cells of the molecular layer of the cerebellum (Fig. 6BC). Because it was difficult to detect OPN3-mCh expression in terminals and GAD65, the other enzyme that catalyzes the conversion of glutamate to GABA is generally concentrated in axon terminals (Kaufman et al., 1991), we could not evaluate the overlap between GAD65 and OPN3-mCh. Future work investigating the overlap of all GABAergic neurons, identified by GAD65 and GAD67 immunoreactivity, with OPN3 expression may be beneficial to fully understand the functions of OPN3. 

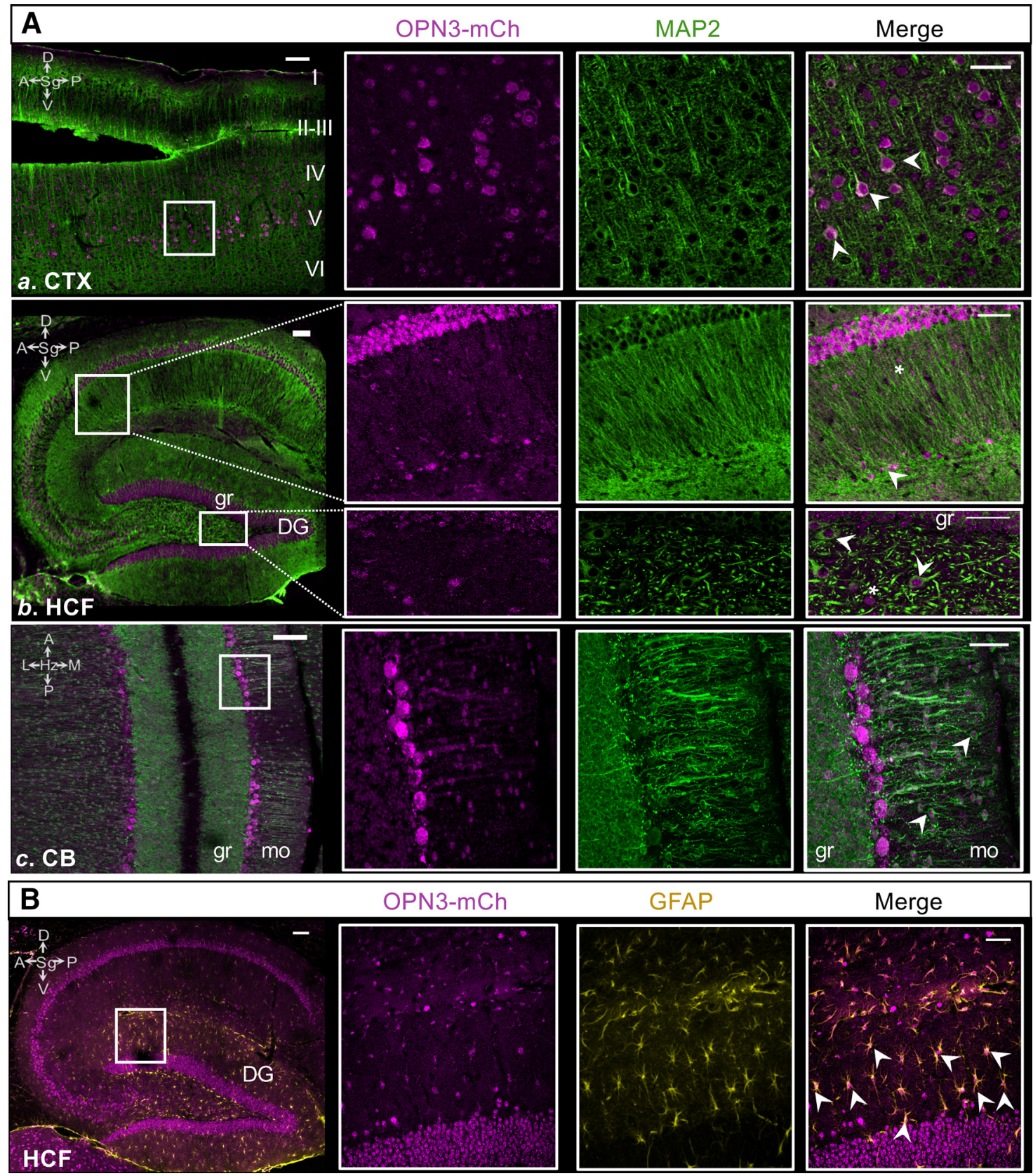

Figure 5. Relative distribution of OPN3 and neuronal (MAP2) or astrocytic (GFAP) markers in the adult OPN3-mCh mouse brain. $\boldsymbol{A}$, Colocalization of OPN3-mCh (magenta) and MAP2 (green). Aa, Large, pyramidal neurons in Layer V of the CTX express OPN3-mCh and the neuronal marker MAP2. $\boldsymbol{A} \boldsymbol{b}$, In the HCF, OPN3-mCh-expressing cells overlap with MAP2 in the granule layer (gr) and in some larger cells at the junction of CA1 and DG (arrowhead). Some smaller, multidendritic OPN3-mCh cells appear not to contain MAP2 (asterisk). Ac, Purkinje cells in the cerebellum (CB) coexpress both MAP2 and OPN3-mCh; OPN3$\mathrm{mCh}$ is present in some granule neurons ( $\mathrm{gr}$ ) and many molecular layer (mo) neurons (arrowheads). B, Colocalization of OPN3mCh (magenta) and GFAP (yellow) in the HCF. Many OPN3-mCh-expressing cells in the HCF with star-like morphology are also immunostained for GFAP, indicating that especially in the DG, astrocytes express OPN3-mCh (arrowheads). Initial zoomed out images for each region have scale bars: $100 \mu \mathrm{m}$. All magnified boxed regions are maximal projections from z-stacks with scale bars: $50 \mu \mathrm{m}$.

\section{Endogenous OPN3-mCh expression in individual brain regions}

To further examine expression patterns of OPN3-mCh and the morphology of these cells, we next looked at OPN3-mCh expression by region.

\section{Olfactory area, CTX, and striatum}

There was robust and dense OPN3-mCh expression in several regions of the olfactory bulb. We observed that the accessory olfactory bulb (AOB) granular layer expressed high levels of OPN3-mCh (Fig. 7A). Within the 

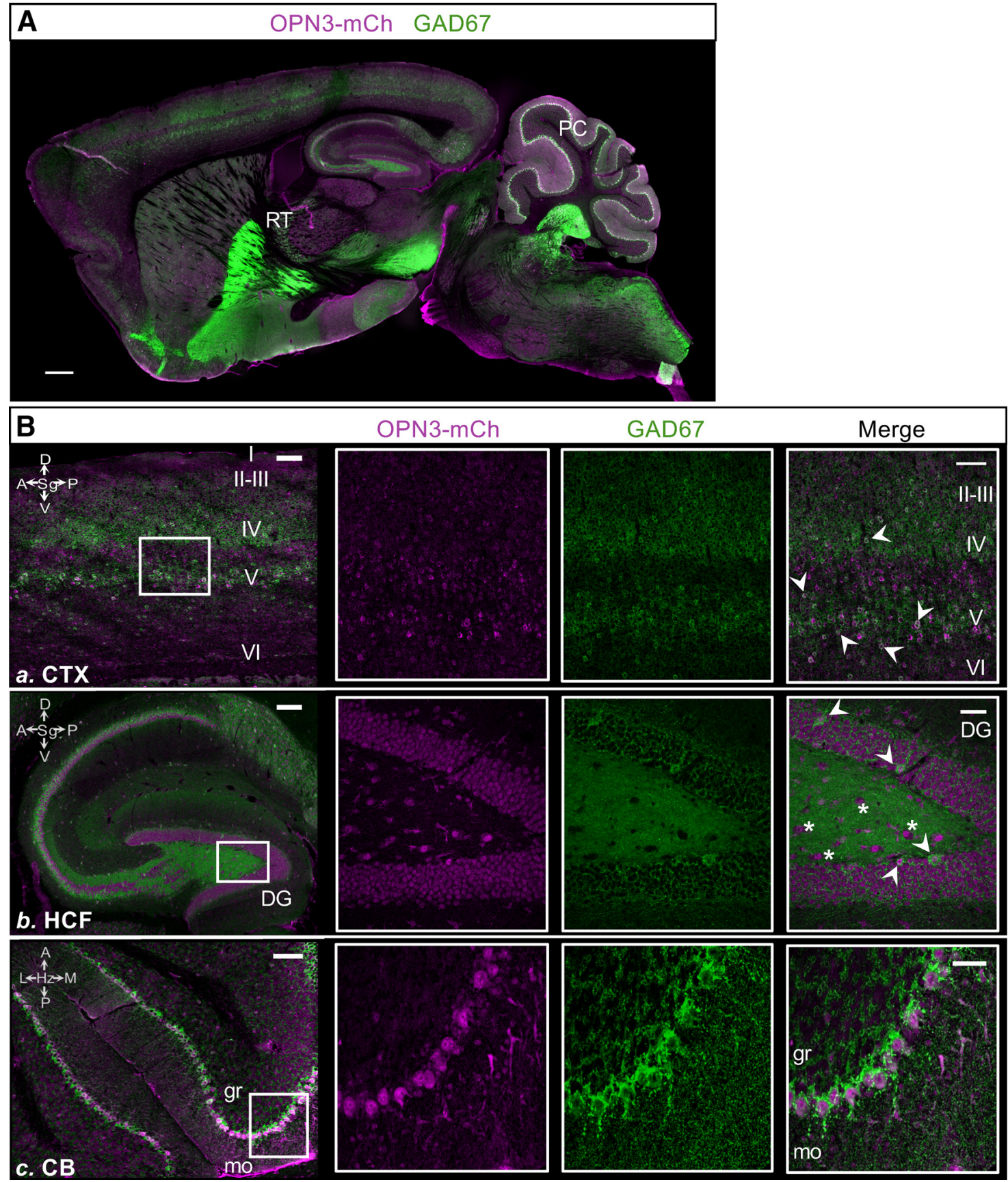

Figure 6. Relative distribution of OPN3 and GAD67 in the adult OPN3-mCh mouse brain. $\boldsymbol{A}$, Distribution of OPN3-mCh (magenta) and GAD67 (green) in a representative sagittal section of the adult OPN3-mCh brain. Scale bar: $500 \mu \mathrm{m}$. PC: Purkinje cell layer of the cerebellum, RT: reticular nucleus of the thalamus. B, Colocalization of OPN3-mCh and GAD67. Ba, Many OPN3-mCh cells are GAD67+ in Layers IV-V and at the V-VI border (arrowheads) of the CTX. Bb, In the HCF, nearly all GAD67+ cells also express OPN3-mCh (arrowheads). But, especially in the DG, not all OPN3 mCh cells are GAD67+ (asterisks). Bc, Purkinje cells are GAD67+ and express OPN3-mCh. OPN3-mCh is present in some, but not all GAD67+ cells within the granule (gr) and molecular (mo) layers of the cerebellum (CB). Initial zoomed out images for each region have scale bars: $100 \mu \mathrm{m}(\boldsymbol{a}, \mathrm{CTX}), 200 \mu \mathrm{m}(\boldsymbol{b}, \mathrm{HCF}), 100 \mu \mathrm{m}$ (c, CB). All magnified boxed regions are maximal projections from zstacks with scale bars: $50 \mu \mathrm{m}$.

main olfactory bulb, OPN3-mCh was expressed predominantly in the mitral cell layer along with a dispersed expression pattern in the external plexiform layer and around the glomeruli (Fig. 7Ab). It was evident under higher magnification that there was a subset of OPN3-mCh+ cells within the granule layer (Fig. 7Abi). In addition to the olfactory bulb, OPN3-mCh was also expressed in piriform area, Layer II (Figs. 2-4, 7Bb).

Previous reports have indicated that OPN3 mRNA (Blackshaw and Snyder, 1999) and protein (Nissilä et al., 

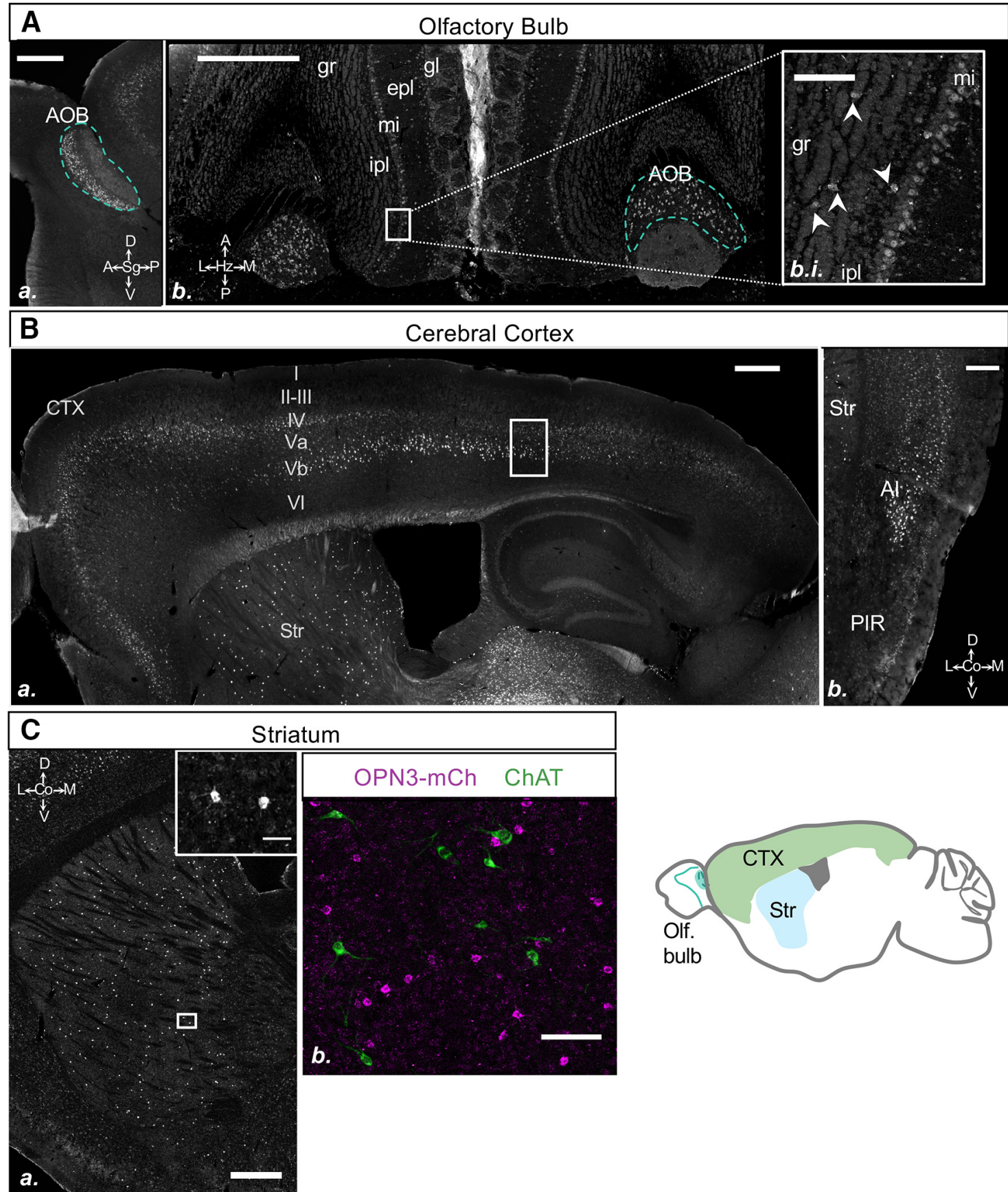

Figure 7. OPN3 expression in the olfactory bulb, CTX, and striatum of the adult OPN3-mCh mouse brain. Aa, There is high OPN3mCh expression in the AOB granular layer (outlined in turquoise). $\boldsymbol{A} \boldsymbol{b}$, OPN3-mCh expression in the AOB, and mitral cell (mi), glomerular (gl), granule (gr), and external plexiform (epl) layers; almost no expression in inner plexiform layer (ipl). Abi, Magnified image of the boxed region of the mitral cell layer in $\boldsymbol{A} \boldsymbol{b}$. Arrowheads denote OPN3-mCh expression in certain cells of the granule (gr) as well as many cells of the mitral (mi) layers. Scale bars: $500 \mu \mathrm{m}(\boldsymbol{A a}, \boldsymbol{A} \boldsymbol{b})$ and $100 \mu \mathrm{m}(\boldsymbol{A} \boldsymbol{c})$. Ba, Expression of OPN3-mCh in two distinct layers of the CTX. Layers III-VI contain OPN3-mCh with the strongest expression in Layers IV and Vb. Bb, Strong OPN3-mCh expression is present in the agranular insular area (AI) and piriform area (PIR), Layer II. Scale bar: $250 \mu \mathrm{m}$. Nissl counterstain is shown in Extended Data Figure 7-1. Ca, OPN3-mCh expression in a subset of small, dendritic cells in the striatum (Str). Scale bar: $500 \mu \mathrm{m}$. Inset, OPN3-mCh cells have several proximal dendrites. Scale bar: $60 \mu \mathrm{m}$. $\mathbf{C b}$, No overlap between ChAT positive cells (green) and OPN3-mCh-expressing cells (magenta) in the Str. Scale bar: $100 \mu \mathrm{m}$.

2012) are expressed exclusively in Layers IV-VI of the CTX. We saw a similar pattern of OPN3-mCh expression with Layers IV-V having the most prominent expression, although we did observe sparse and weak expression of
OPN3-mCh in Layers I-III as well as VI (Fig. 7Ba). Expression was highest in the frontal and cingulate cortices when viewed horizontally (Fig. $2 B$ ). It was clear from a sagittal view that OPN3-mCh exhibited a double-banded 

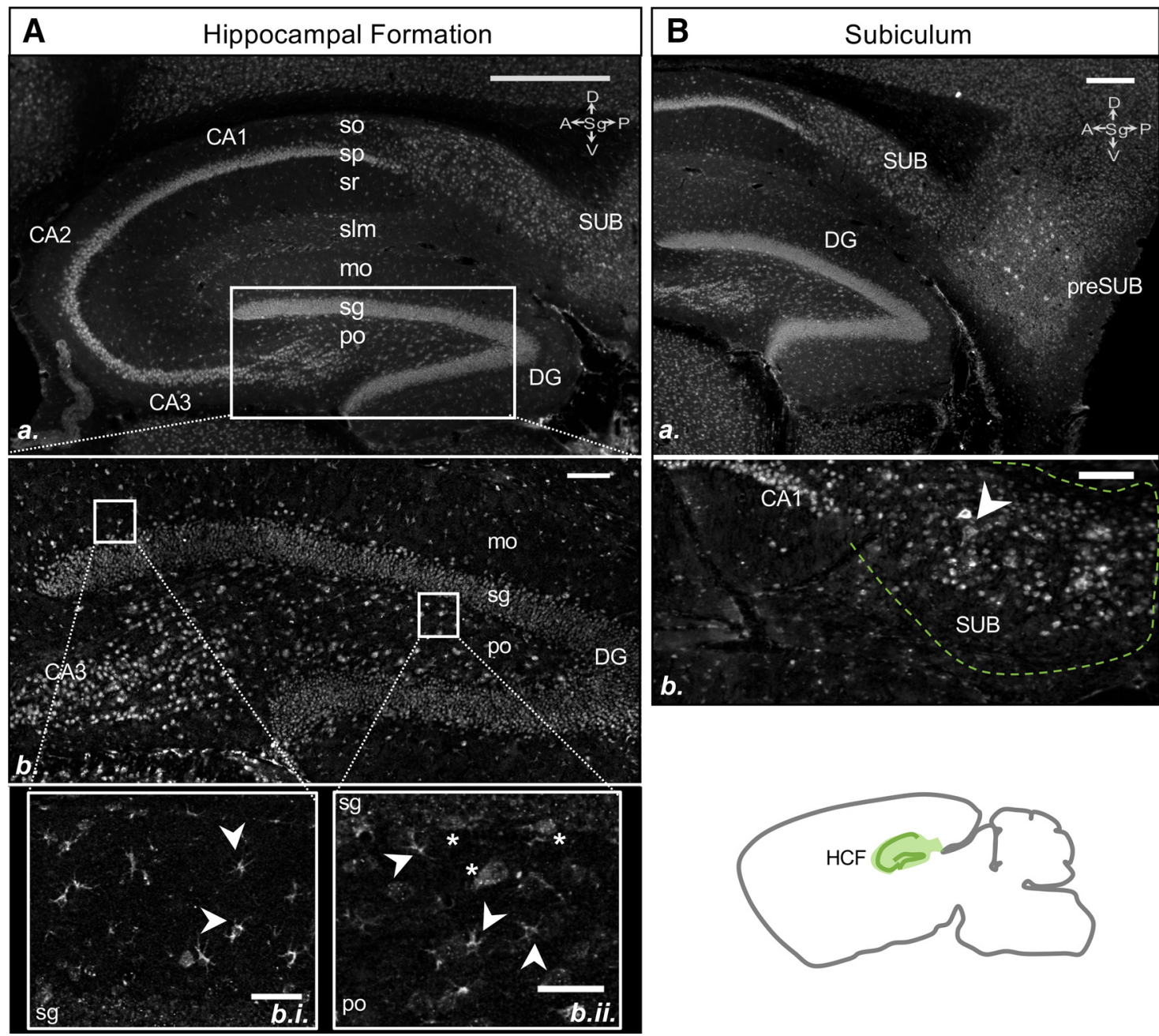

Figure 8. OPN3 expression in the HCF of the adult OPN3-mCh mouse brain. $\boldsymbol{A a}$, OPN3-mCh in the HCF. Scale bar: $500 \mu \mathrm{m}$. $\boldsymbol{A} \boldsymbol{b}$, OPN3-mCh in a variety of cell types in all layers of the DG. Scale bar: $100 \mu \mathrm{m}$. Abi, Magnified image of DG-mo to DG-sg regions with highly dendritic OPN3-mCh+ cells (with astrocyte-like morphology) indicated by arrowheads. Scale bar: $50 \mu \mathrm{m}$. Abii, Magnified image of DG-sg to DG-po regions with highly dendritic astrocytes indicated by arrowheads and larger, triangular neurons indicated by asterisks. Scale bar: $50 \mu \mathrm{m}$. Ba, Expression of OPN3-mCh in the subiculum (SUB) and presubiculum (preSUB). Scale bar: $250 \mu \mathrm{m}$. B b , OPN3-mCh expression at the plasma membrane and in some proximal dendrites of large cells in the SUB (arrowhead). Scale bar: $100 \mu \mathrm{m}$. Layers: mo: molecular, sg: granule, po: polymorph, slm: stratum lacunosum-moleculare, so: stratum oriens, sp: pyramidal cell, sr: stratum radiatum.

pattern with robust expression in Layer IV and in large pyramidal cells of Layer $\mathrm{Vb}$ as sections progressed from lateral to more medial (Fig. 7B; see also Fig. 2A). OPN3-mCh expression extended from agranular insular, orbital, somatomotor to somatosensory areas where double banding became pronounced through posterior parietal associated areas, with expression decreasing caudally through the visual areas and retrosplenial area. The gradient of decreasing expression along the rostrocaudal axis was also observed in coronal sections, where it became evident that the highest OPN3-mCh expression was in the motor and insular areas with diminishing expression as the brain progressed caudally into the auditory and temporal areas. Interestingly, despite decreasing OPN3-mCh expression in the caudal CTX $\sim-2 \mathrm{~mm}$ posterior to bregma, there was continued, robust, OPN3-mCh expression in Layer $\mathrm{V}$ of the agranular insular and perirhinal areas (Figs. 3-4, 7Bb).
When it was first discovered, OPN3 mRNA was detected in small cells within the striatum of the mouse (Blackshaw and Snyder, 1999). Since then, a recent publication investigated OPN3 expression in the macaque striatum (El Massri et al., 2018). This report identified two distinct populations of OPN3-expressing interneurons in the striatum: giant cholinergic interneurons positive for ChAT and smaller, ChAT-negative cells. A larger population of ChAT+ interneurons contained OPN3 than the ChAT-negative interneurons. To evaluate whether OPN3 expression in the mouse was similar to what has been observed in the monkey, we examined the striatum of the OPN3-mCh mouse more closely. We observed strong OPN3-mCh expression in small cells, evenly distributed throughout the striatum (Fig. $7 \mathrm{Ca}$ ). mCh immunoreactivity in these cells was not limited to the soma but was also present in many proximal processes. Unlike in the 

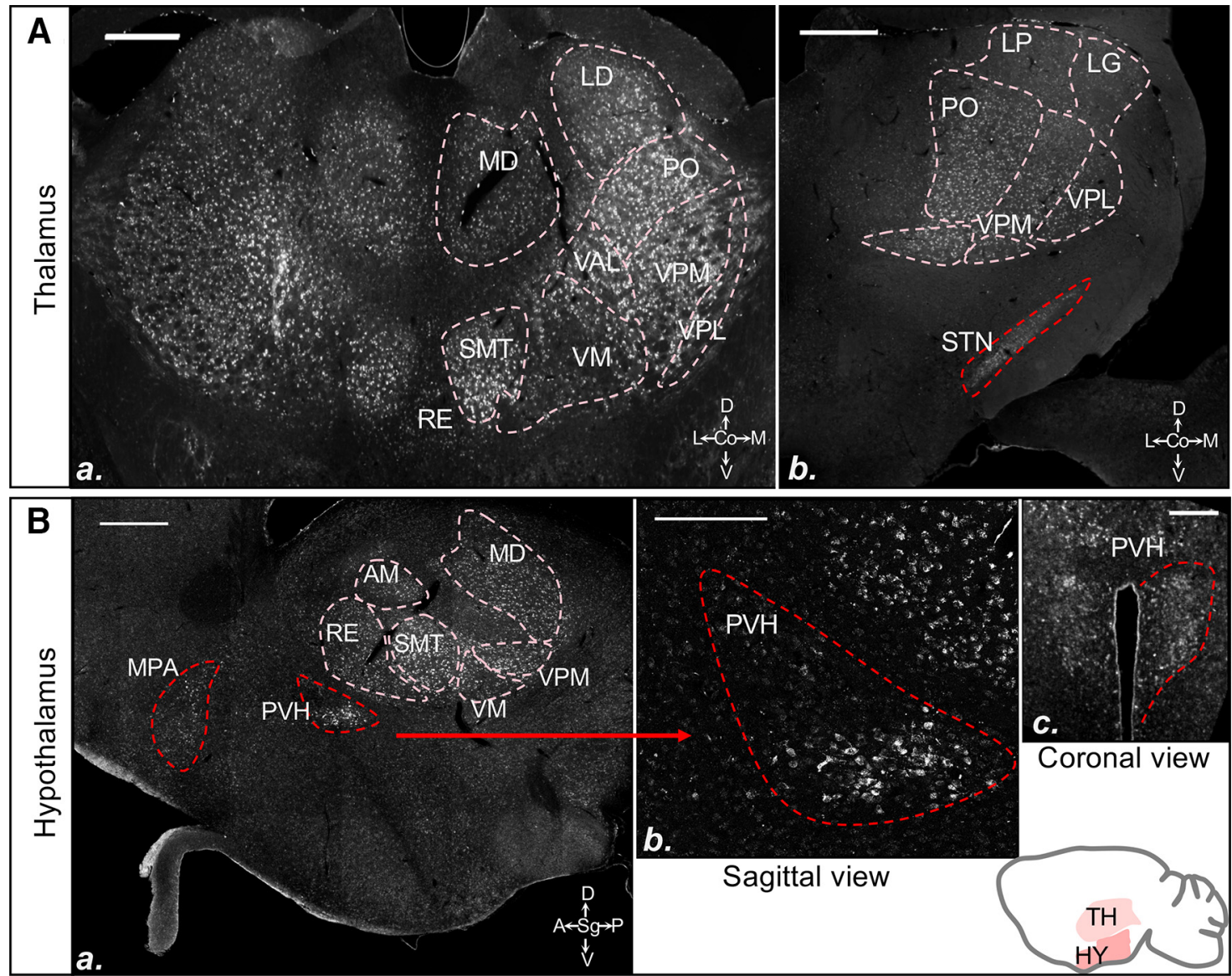

Figure 9. OPN3 expression in the thalamus and hypothalamus of the adult OPN3-mCh mouse brain. Aa, High OPN3-mCh expression in the thalamus (TH), especially in ventromedial and lateral nuclei with moderate expression in the nucleus of reuniens (RE) and mediodorsal nucleus (MD) and low expression in the anteromedial nucleus (AM). A $\boldsymbol{b}$, High expression of OPN3-mCh is also detected in the subthalamic nucleus (STN). Scale bars: $500 \mu \mathrm{m}$. Ba, Sagittal view of the thalamus and hypothalamus (HY) shows high OPN3-mCh expression in the PVH and moderate expression in the MPA of the hypothalamus. Scale bar: $500 \mu \mathrm{m}$. Bb, High density of OPN3-mCh-expressing cells in the PVH. Bc, Coronal view of OPN3-mCh expression in both hemispheres of the PVH. Scale bars: $200 \mu \mathrm{m}(\boldsymbol{B} \boldsymbol{b}, \boldsymbol{B} \boldsymbol{c})$. Pink outline: thalamic nuclei; red outline: hypothalamic nuclei. Nissl counterstains can be found in Extended Data Figure 7-1.

monkey striatum, however, we did not observe OPN3 colocalization with ChAT (Fig. 7Cb). OPN3-mCh-expressing cells were smaller in diameter than ChAT-expressing cells, and we saw no indication that OPN3-mCh is expressed in giant cholinergic interneurons in the mouse striatum. This discrepancy is likely because of species differences: monkeys and other more evolved organisms have a much larger striatum than rodents (Petryszyn et al., 2018), and the mouse has a smaller quantity of ChAT+ interneurons. Over evolutionary time, OPN3 may have developed specific functions in ChAT+ interneurons of higher organisms.

\section{$\mathrm{HCF}$}

Of the previous reports exploring OPN3 in the mouse brain, none have closely examined OPN3 expression in the HCF. Using the OPN3-mCh mouse, we consistently found high expression of OPN3-mCh in the HCF; OPN3-mCh was present in the presubiculum, subiculum, CA1/2/3, and DG (Fig. 8).

Within CA1/2/3, OPN3-mCh was apparent in or around the pyramidal cell layers. Within the stratum oriens, there were large, intensely labeled cells with little dendritic staining along with smaller, extensively branched cells with $\mathrm{mCh}$ immunoreactivity in both the soma and dendrites. These two populations were also observed in the stratum radiatum and stratum lacunosum-moleculare, with a higher density of OPN3-mCh in smaller, branched cells within the stratum lacunosum-moleculare than the other stratum layers (Fig. 8Aa). Within the DG, OPN3-mCh was observed among all layers: molecular, granule, and polymorph. OPN3-mCh expression in the molecular layer consisted of small, star-like cells throughout (GFAP+ astrocytes; see Fig. $5 B$ ) and expression in larger, moderately branched cells immediately proximal to the granule layer (Fig. 8Abi). The polymorph layer had substantial expression of OPN3-mCh; the morphologies of the OPN3-mCh + cells indicated that there were different populations expressing OPN3-mCh. Larger, triangular shaped cells at the boundary of the granule and polymorph layers are likely the cell bodies of dentate pyramidal basket cells, which coimmunostain for GAD67 (Figs. $8 A b$, Abii; see also Fig. 6Bb). There were also 
A
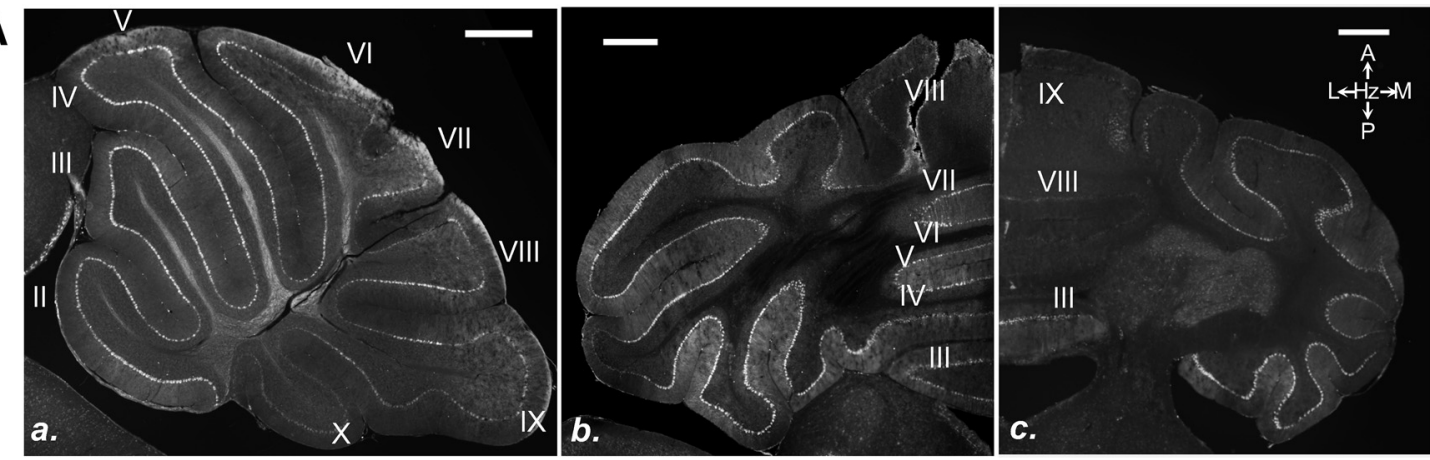

B
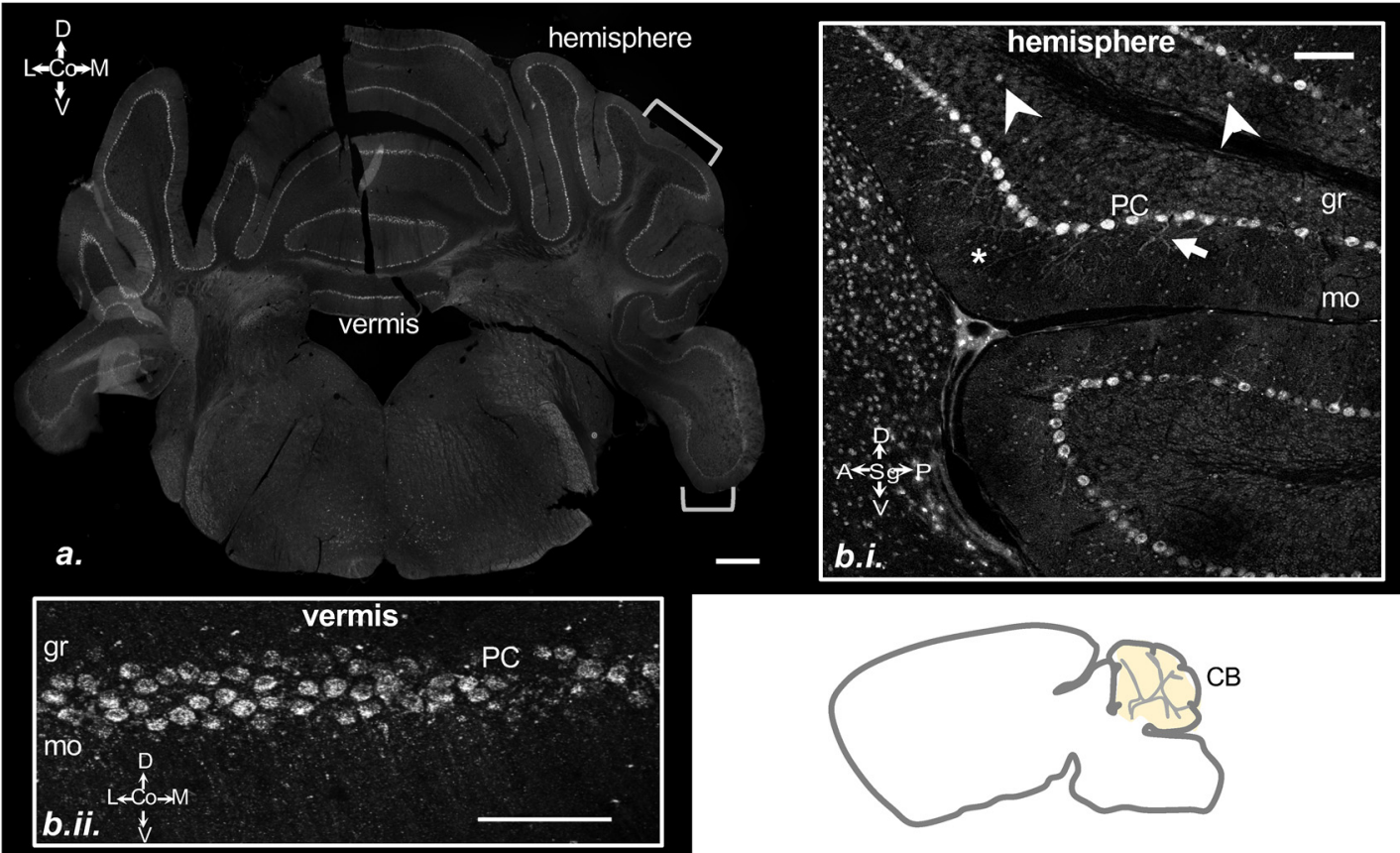

Figure 10. OPN3 expression in the cerebellum of the adult OPN3-mCh mouse brain. Aa, OPN3-mCh expression in a sagittal view of the cerebellum (CB). Lobes are labeled with numerals. OPN3-mCh expression is strongest in Lobes II-VI and decreasingly lower in VII-X. $\boldsymbol{A} \boldsymbol{b}, \boldsymbol{A c}$, Horizontal serial sections of the cerebellum with $(\boldsymbol{A} \boldsymbol{b})$ more rostral than $(\boldsymbol{A} \boldsymbol{c})$. OPN3-mCh expression decreases as the cerebellar lobes progress, with nearly negligible expression in Lobes VIII and IX. Scale bars: $500 \mu \mathrm{m}$. B, A representative coronal section of the cerebellum with areas containing little to no OPN3-mCh expression (brackets) and areas with significant OPN3-mCh expression. Scale bar: $500 \mu \mathrm{m}$. Bbi, Magnified sagittal image of the hemisphere showing OPN3-mCh in the granule layer (gr; arrowheads) and to a greater extent in the molecular layer (mo; asterisks). OPN3-mCh can be observed in the processes of the Purkinje cells (PC; arrow). Scale bar: $100 \mu \mathrm{m}$. Bbii, Magnification of the Purkinje cell layer in the vermis of a coronal section. Scale bar: $100 \mu \mathrm{m}$.

smaller cells with varying degrees of dendritic arborization. Based on coimmuno-staining with GAD67, some appeared to be inhibitory while others that were not stained for GAD67 may instead be mossy cells (Fig. $8 A$ bii; see also Figs. $5 B, 6 B b$ ). Within the subiculum and presubiculum, OPN3-mCh expression was robust in a subset of cells (Fig. 8B).

\section{Thalamus and hypothalamus}

OPN3 mRNA was shown to be highly expressed in the mouse thalamus with a particularly high density in lateral regions (Blackshaw and Snyder, 1999). We saw a consistent pattern, with the thalamus being one of the most OPN3-enriched regions of the brain (Fig. 2). Upon closer inspection, we found OPN3-mCh primarily expressed in ventral, lateral, mediolateral and posterior nuclei with little to no expression in more central regions. Figure $9 A$ shows the striking pattern of ventrolateral OPN3-mCh expression. OPN3-mCh was present in the lateral dorsal (LD), lateral geniculate (LG), lateral posterior (LP), posterior (PO), submedial (SMT), ventral posterolateral (VPL), ventral medial (VM), ventral lateral (VAL) and ventral posteromedial (VPM) nuclei. Highest OPN3-mCh expression was observed in the SMT, VAL, VPL, and VPM (Fig. 4).

In the hypothalamus, OPN3 mRNA was initially identified in the PVH and medial preoptic area (MPA) by RNA-seq (Dutra et al., 2020), Western blotting, and immunostaining (Nissilä et al., 2012). We confirmed that OPN3-mCh was expressed in the MPA and highly expressed in the PVH (Fig. 9B). RNA-seq has suggested that OPN3 is present in the supraoptic nucleus (Dutra et al., 2020), a region where we also found high OPN3-mCh expression. Additionally, 


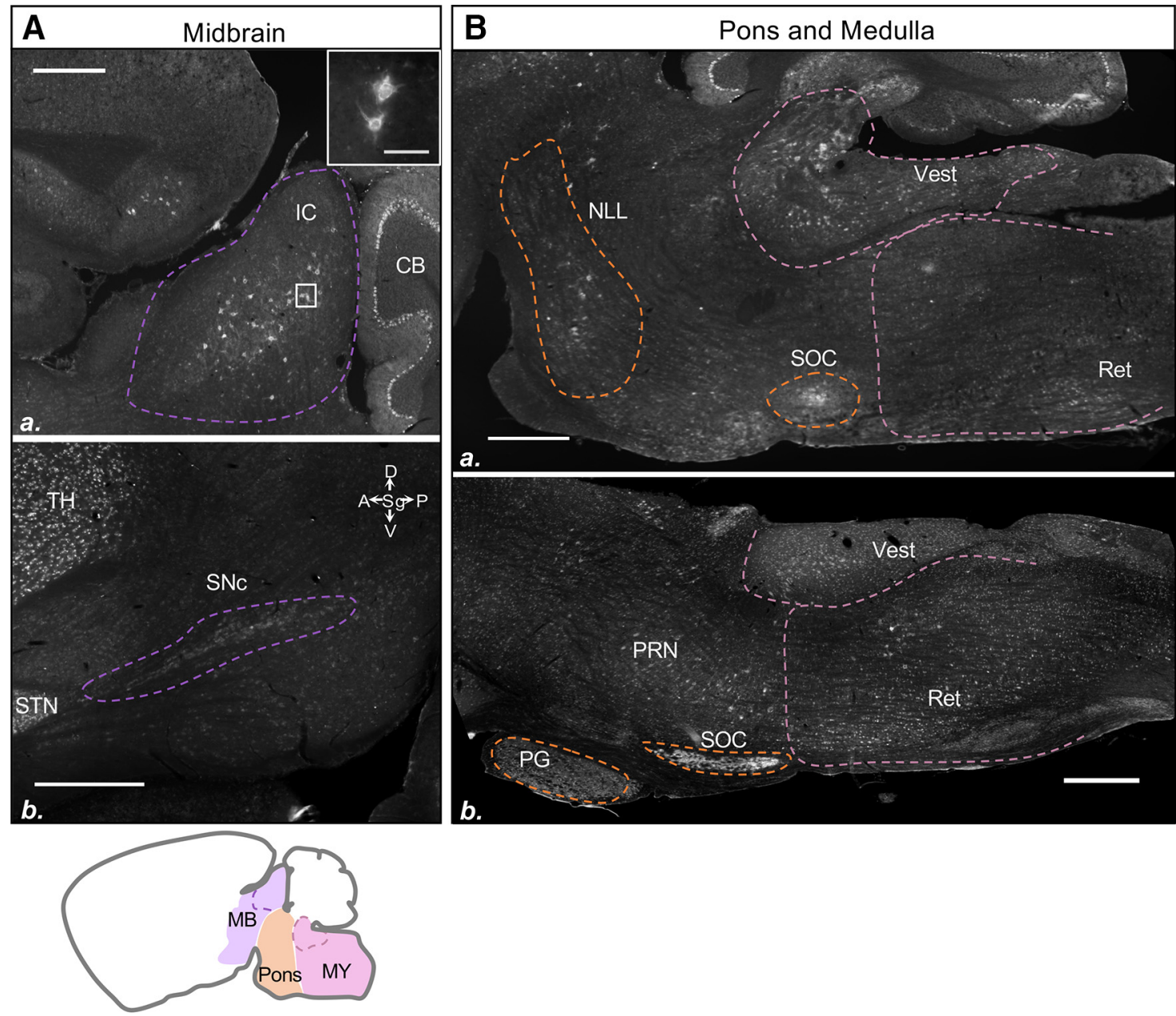

Figure 11. OPN3 expression in the midbrain, pons and medulla of the adult OPN3-mCh mouse brain. Aa, Robust OPN3-mCh expression in the inferior colliculus (IC) by the cerebellum (CB). Scale bar: $500 \mu \mathrm{m}$. Inset, Higher magnification boxed region shows multidendritic branching of the OPN3-mCh + cells. Scale bar: $100 \mu \mathrm{m}$. $\boldsymbol{A} \boldsymbol{b}$, OPN3-mCh shows distinct expression in the substantia nigra compacta (SNc) posterior to the subthalamic nucleus (STN). Scale bar: $500 \mu \mathrm{m}$. Ba, Widefield image of the pons and medulla with regions of higher OPN3-mCh expression outlined. $\mathbf{B b}$, A more medial section of the pons shows pontine reticular nucleus $(\mathrm{PRN})$, pontine gray (PG), and superior olivary complex (SOC). Scale bars: $500 \mu \mathrm{m}(\mathbf{B a}, \mathbf{B b})$. Vest: vestibular nuclei, Ret: reticular nuclei NLL: nucleus of the lateral lemniscus. Purple outline: midbrain (MB), orange outline: pons, pink outline: medulla (MY). Nissl counterstain can be found in Extended Data Figure 7-1.

we saw high OPN3-mCh expression in the subthalamic nucleus (Fig. 9Ab; see also Fig. 2A). Lower expression was identified in the arcuate nucleus, dorsomedial nucleus, and lateral mammillary nucleus (Fig. 4).

\section{Cerebellum}

Albeit consisting of only three studies, OPN3 expression in the cerebellum is the most widely studied of all OPN3-expressing regions (Blackshaw and Snyder, 1999; Lein et al., 2007; Nissilä et al., 2012). OPN3 mRNA was previously found in the Purkinje cell layer of both the vermis and hemispheres of the cerebellar cortex. Within the Purkinje layer, OPN3 mRNA follows a rostrocaudal gradient, having low to no expression within Purkinje cells in more caudal Lobes VIII-X. This gradient was only observed in mice greater than postnatal day 20 and was most prominent in the adult cerebellum (Blackshaw and Snyder, 1999). This gradient was not examined in the
Nissilä et al. (2012) study, and it was unknown whether OPN3 protein followed the same pattern of expression. We examined the cerebellum from coronal, sagittal, and horizontal planes and confirmed that the gradient observed for OPN3 mRNA is conserved for the OPN3-mCh protein. In the adult OPN3-mCh mouse, we saw OPN3$\mathrm{mCh}$ in the Purkinje cell layer decrease in expression in more caudal lobes (Fig. 10A). OPN3-mCh was present in the Purkinje layer of the vermis and both hemispheres, with strong $\mathrm{mCh}$ immunoreactivity in both the soma and proximal dendrites (Fig. 10Bbi). In addition, OPN3-mCh was also expressed in a subset of cells within the granule layer and in many neurons of the molecular layer (Fig. $10 B$; see also Figs. $5 A c, 6 B c)$. In the Blackshaw and Snyder (1999) and Lein et al. (2007) studies, OPN3 mRNA was observed to follow a radial striped pattern in the form of sharp delineated bands of expression across the vermis and hemispheres. We could not identify such marked 
banding patterns for OPN3-mCh protein expression, but, as in Lein et al. (2007), we observed that OPN3-mCh expression was not as intense at the outer edge of Lobe VI and in the paraflocculus (Fig. 10Ba).

\section{Midbrain, pons, and medulla}

The only previous investigation into OPN3 expression in the midbrain and hindbrain reported that embryonic day 18.5 mice have substantial levels of OPN3 mRNA in the dorsal pons (Blackshaw and Snyder, 1999). We sought to examine if this substantial expression persists in the adult OPN3-mCh mouse brain. We found that OPN3-mCh is only sparsely expressed in the adult midbrain and hindbrain. Within the midbrain, we discovered strikingly robust OPN3-mCh expression in the inferior colliculus among a subset of multidendritic cells (Fig. 11Aa). Less robust was OPN3-mCh expression in the substantia nigra compacta (Fig. 11Ab). In the pons, there was sparse expression in the nucleus of the lateral lemniscus and throughout the pontine reticular nuclei, moderate expression in the pontine gray, and high expression in the superior olivary complex (Fig. 11B). The medulla showed moderate OPN3$\mathrm{mCh}$ expression dispersed throughout the vestibular and reticular nuclei, particularly in the medial vestibular nucleus directly below the cerebellum and the magnocellular reticular nucleus (Fig. 11B).

\section{Discussion}

While the distribution of encephalic OPN3 mRNA has previously been examined (Blackshaw and Snyder, 1999; Halford et al., 2001; Dutra et al., 2020) and OPN3 expression for some brain regions has been studied using promoter-driven GFP reporter mice (GENSAT; Gong et al., 2003) and immunostaining (Nissilä et al., 2012; El Massri et al., 2018), our analysis of OPN3-mCh expression from coronal, sagittal, and horizontal planes across the adult OPN3-mCh brain has uncovered novel findings. We have first identified new regions in the brain in which OPN3 expression was previously unknown including: mitral cell layer, piriform area and nucleus of the lateral olfactory tract of the olfactory areas; CA regions and presubiculum of the HCF; posterior hypothalamic nucleus; inferior colliculus, and nucleus sagulum of the midbrain; and pontine gray and superior olivary complex in the pons (Extended Data Fig. 4-1, red text). We compared our OPN3-mCh expression with that of previous OPN3 mRNA and protein expression analyses, and in addition to our novel areas, our findings corroborate previous work (Extended Data Fig. 4-1). There were some instances of dissimilarity between our OPN3-mCh study and the OPN3 mRNA and reporter protein analyses, but these could be because of several factors (Extended Data Fig. 4-1, blue text). OPN3 is known to be developmentally regulated as its expression in the CTX, cerebellum, and pons differs from the embryo to adult (Blackshaw and Snyder, 1999). We focused our study only on the adult mouse which may have different expression profiles than neonates analyzed in previous reports. We may also see differences, especially from mRNA expression, due to differences in translational processing between mRNA and protein levels. This is explicit as little to no mRNA has been detected in the HCF [Allen Brain Atlas ISH Data (Lein et al., 2007)], yet we and others observe protein expression in the DG (Gong et al., 2003). We see the most differences between our OPN3mCh expression mapping and that of the GENSAT OPN3GFP reporter mouse. A major drawback to GFP reporters is the lack of endogenous degradation and turnover of the exogenous GFP, especially when OPN3 is developmentally regulated. In addition, BAC-based GFP reporters could be leaky and generate false positives. These potentially confounding factors are eliminated in our OPN3-mCh model in which the reporter fluorophore is fused to OPN3. The OPN3-mCh mouse also gives the ability to discern developmental regulation because OPN3-mCh is presumably degraded as the endogenous protein would be.

Additionally, we discovered that OPN3 is expressed not only in neurons but also in astrocytes within the HCF. This is the first indication of OPN3 in astrocytes. We are also the first to characterize horizontal sections which, in addition to coronal and sagittal sections, allowed us to uncover the GABAergic nature of many OPN3 cells, determine the non-ChAT nature of OPN3 cells in the striatum, and reveal of OPN3 subcellular localization in the soma and some cell terminals.

We find that OPN3 is expressed throughout the adult brain, but it displays differential distribution (Figs. 2-4). One unifying factor among all regions of OPN3-mCh expression is the presence of cytoplasmic OPN3-mCh predominantly in the soma with little to no immunostaining in processes. This is consistent with previously reported cellular distribution of OPN3 protein, albeit the immunostaining resolution is poor in the reported images (Nissilä et al., 2012). In some instances, however, especially larger cells with thicker dendrites, we do observe OPN3-mCh in regions most proximal to the soma; as processes extend radially from the cell, OPN3-mCh expression fades [see CTX pyramidal neurons (Fig. 7A); Purkinje cells (Fig. 10B); inferior colliculus (Fig. 11A)]. This could indicate that OPN3-mCh is expressed only at low levels in processes and with smaller cells, the combination of thinner processes and low OPN3-mCh expression makes OPN3 detection difficult. It is unlikely that the $\mathrm{mCh}$ tag used for the OPN3-mCh mice would adversely affect or limit endogenous OPN3 distribution to cell processes. This was a major concern of ours even before generating the OPN3$\mathrm{mCh}$ mouse model and we took precautions to the best of our ability to ensure that OPN3-mCh maintained endogenous localization. In our previous publication, we identified that OPN3 subcellular localization in melanocytes and other heterologous cell types is at the plasma membrane, as expected of a GPCR, but also in internal vesicles within the cytoplasm; OPN3-mCh significantly colocalized with endogenous OPN3 (using the now discontinued OPN3 antibody) in primary human melanocytes (Ozdeslik et al., 2019). In addition to retaining its subcellular localization, we found that OPN3-mCh retained its regulatory activity in melanocytes, suggesting that OPN3$\mathrm{mCh}$ is functional despite fusion to a fluorescent protein (Ozdeslik et al., 2019). Although there is a possibility that OPN3 processing may lead to altered subcellular 
localization of OPN3-mCh, we believe this is unlikely, as tagging other GPCRs at the direct C terminus with fluorescent proteins and using similar knock-in methods did not alter subcellular localization (as in the MC4R-GFP mouse; Siljee et al., 2018). We can also confidently exclude the possibility that $\mathrm{mCh}$ is cleaved after protein processing as $\mathrm{mCh}$ is a small $(\sim 27 \mathrm{kDa})$, soluble protein with cellular localization very different from that of the larger OPN3-mCh ( $\sim 49 \mathrm{kDa})$, with seven transmembrane domains typical of all GPCRs; based on its size and topology, mCh localizes within the somas and terminals of the cells (Chen et al., 2013), whereas we consistently observe a punctate or granular appearance to the OPN3mCh staining (Fig. 5, magnified images as examples), consistent with a membrane protein expressed at the plasma membrane and in membrane-bound intracellular compartments.

Alternatively, particularly in the HCF where OPN3-mCh appeared to be expressed within somas and minimally in dendrites in the DG granule and CA1/2/3 pyramidal cell layers (Figs. 5, 6Bb, 8), it is possible that some of the apparently somatic staining is actually staining of axon terminals that innervate these layers. The pattern of punctate OPN3-mCh staining, especially around the pyramidal cell and the granule layers, could be consistent with high OPN3-mCh expression in the axon terminals of GABAergic interneurons innervating the pyramidal and granule cells (Freund and Buzsáki, 1998) and not solely OPN3-mCh expression in the somas of the pyramidal and granule cells. The present work did not attempt to discriminate between somatic labeling versus innervations of cells by axon terminals, but this may be of interest for future studies.

Taken as a whole, OPN3-mCh expression patterns broadly follow circuitry related to sensorimotor pathways. OPN3-mCh has notably high expression in the olfactory areas including the $\mathrm{AOB}$, piriform area, olfactory tubercle and many layers of the main olfactory bulb. It is well known that cortico-thalamo-cortical pathways are essential in the processing of most sensations, among a myriad of other functions (Guillery and Murray Sherman, 2002). OPN3-mCh is present in the somatosensory areas of the CTX which form connections with the hub of sensory circuitry, the thalamus, the region where OPN3-mCh is most highly expressed. The ventral posterior nuclei of the thalamus where OPN3-mCh expression is prominent, aids in processing somatosensory input, among other functions (Pierret et al., 2000; Bureau et al., 2006; Wimmer et al., 2010; Ohno et al., 2012; Oh et al., 2014). OPN3 is also expressed in the mouse retina (Nissilä et al., 2012; Buhr et al., 2015), possibly explaining OPN3-mCh expression in the LGd of the thalamus, which is the terminal region of most retinal ganglion cells (Szentagothai, 1963). OPN3$\mathrm{mCh}$ is furthermore expressed in many regions essential for the relay and processing of auditory information: auditory cortex, medial geniculate complex of the thalamus, inferior colliculus and superior olivary complex of the midbrain and hindbrain, respectively (Oh et al., 2014; Blazquez Freches et al., 2018). We also found OPN3-mCh in the motor nuclei of the thalamus, the motor area of the
CTX, Purkinje cells, and other nuclei of the cerebellum and in ventral pontine nuclei.

In addition to possibly being involved in larger, systemslevel functions like sensation and motor control, it is likely that OPN3 performs region-specific functions in a variety of different cell types (neurons and GFAP + cells) that may, or may not, work together toward a unifying role. For example, we have shown that OPN3-mCh is expressed in GABAergic Purkinje cells of the cerebellum and GABAergic cells in the DG (Fig. 6), yet it is also expressed in the excitatory pyramidal and granule cells of the HCF and excitatory cells of the thalamus (Figs. 8, 9). It is evident that OPN3 does not simply follow a neuronal phenotype-specific pattern but is regulated in a more complex region-specific manner and even within the same brain region by different inputs and microdomains. For instance, the most intriguing and unanticipated expression of OPN3-mCh was in the HCF. In the HCF, OPN3-mCh is surprisingly abundant given that previous mRNA reports (Allen Brain Atlas ISH Data) have indicated no expression in this area (Lein et al., 2007). As mentioned, differences in mRNA regulation and translation into protein may account for the differences. There is one other report from the GENSAT database of OPN3 being highly expressed in the granule layer of the DG, but no further studies examined HCF protein expression (Gong et al., 2003). This is the only region in which we readily observe star-like, GFAP+ astrocytic OPN3-mCh immunoreactivity in addition to its presence in neurons. Although astrocytes are present throughout the brain, they, like neurons, have different morphotypes, respond to different stimuli, and have different functions (for review, see Miller, 2018). Even within the HCF itself, astrocytes exhibit differences between the CA layers and the DG layers (Matyash and Kettenmann, 2010; Jinno, 2011). Indeed, it has been shown using DREADDs and endogenous receptors that astrocytes of the hippocampal stratum radiatum (as opposed to the striatum) have differential Gi-coupled GPCR signaling that may result from variation in gene expression (Chai et al., 2017). Because OPN3 in a variety of species, including human, has been shown to couple to the G-protein $\alpha$-subunit Gi (Koyanagi et al., 2013; Sugihara et al., 2016; Ozdeslik et al., 2019), OPN3 could exert specific Gi-based signaling or regulation specific to the HCF, explaining its strong expression in astrocytes in the HCF versus other regions.

OPN3, as a member of the opsin family of light-receptive GPCRs, may also participate in light-evoked processes in the brain. OPN3 homologs in mosquito, pufferfish, zebrafish, and chicken absorb blue light (Koyanagi et al., 2013; Sugihara et al., 2016). Human OPN3, however, does not absorb visible light by UV-visible spectroscopy (Ozdeslik et al., 2019), despite several studies indicating OPN3-meditated blue light responses in mammals (Kato et al., 2016; Buscone et al., 2017; Barreto Ortiz et al., 2018; Regazzetti et al., 2018; Yoshimoto et al., 2018; Castellano-Pellicena et al., 2019; Nayak et al., 2020; Sato et al., 2020). In lower vertebrates, photosensitive areas in the diencephalon contain many light-responsive pigments and opsins that directly mediate physiological reactions like color change and motor responses independent of the eyes or pineal glands (Van Veen et al., 1976; Tabata, 1982). It has been demonstrated in a variety of mammalian species from rodents to humans that visible and near infrared light is capable of penetrating 
through the skull into the brain, albeit not very far (Van Brunt et al., 1964; Viggiani et al., 1970; Hartwig and van Veen, 1979; Kobat et al., 2011). Most of the visible light is scattered by bone and tissue, but in smaller animals like rodents, a small percentage of light (red $>$ blue $>$ green; Viggiani et al., 1970) can penetrate into the brain, even to diencephalic regions like the hypothalamus (Van Brunt et al., 1964).

If OPN3 were to act as a photoreceptor, it would require a chromophore, most likely 11-cis retinal, for activation; the brain has a supply of retinoids and intriguingly, RPE65, the enzyme that catalyzes the isomerization of all trans retinyl ester to 11-cis retinol was shown to be expressed in cells lining the third ventricle (Shearer et al., 2010; Ransom et al., 2014), located just ventral to the $\mathrm{PVH}$. We have found OPN3-mCh expression explicitly in the PVH of our knock-in mouse. It is therefore conceivable that OPN3, even in deep diencephalic regions of the mouse brain, has the resources available to act in a photosensitive manner.

Despite the widespread expression of OPN3 shown in this study, the OPN3 knock-out mouse shows no obvious phenotype. Although we can speculate on the potential brain functions of OPN3 based on its expression patterns, the functional roles of OPN3 in the brain remain elusive. It is likely that the OPN3 knock-out mouse has a yet unidentified neurologic phenotype that does not manifest in the conditions or life span to which we house our mice. Indeed, the knock-out mouse was first generated years ago (Buhr et al., 2015), but it has just recently been discovered that only when perturbing normal living and physiological conditions does the OPN3 knock-out mouse exhibit diet-induced obesity and insulin resistance because of dysregulation of metabolic processes in brown adipocytes (Sato et al., 2020) and deficiency in thermogenesis under cold shock because of dysregulation of lipolysis in adipose tissue (Nayak et al., 2020). Interestingly, none of these phenotypes have demonstrated correlations to OPN3 expression in the brain.

While the functional roles of OPN3 cannot be determined solely on its expression pattern, we have made a large step in understanding potential roles for OPN3 in the brain. With the generation of the OPN3-mCh mouse, we have created a novel tool that will further the investigation of OPN3 and will allow for the complete characterization of OPN3 not only in different areas of the brain, but also in all tissues within the mouse. Our model allows the identification of subcellular localization of OPN3 across the entire organism, examination of protein interactions and colocalization without reliance on OPN3 antibodies, and analysis of developmental regulation of the receptor. The generation of the OPN3-mCh mouse model will ignite and facilitate new research into OPN3 function, an area that has lain stagnant for several decades largely because of a lack of reliable and specific identification methods for OPN3 expression.

\section{References}

Asada H, Kawamura Y, Maruyama K, Kume H, Ding RG, Kanbara N, Kuzume H, Sanbo M, Yagi T, Obata K (1997) Cleft palate and decreased brain gamma-aminobutyric acid in mice lacking the 67$\mathrm{kDa}$ isoform of glutamic acid decarboxylase. Proc Natl Acad Sci USA 94:6496-6499.
Barreto Ortiz S, Hori D, Nomura Y, Yun X, Jiang $\mathrm{H}$, Yong $\mathrm{H}$, Chen J, Paek S, Pandey D, Sikka G, Bhatta A, Gillard A, Steppan J, Kim $\mathrm{JH}$, Adachi H, Barodka VM, Romer L, An SS, Shimoda LA, Santhanam L, et al. (2018) Opsin 3 and 4 mediate light-induced pulmonary vasorelaxation that is potentiated by $\mathrm{G}$ protein-coupled receptor kinase 2 inhibition. Am J Physiol Lung Cell Mol Physiol 314:L93-L106.

Blackshaw S, Snyder SH (1999) Encephalopsin: a novel mammalian extraretinal opsin discretely localized in the brain. J Neurosci 19:3681-3690.

Blazquez Freches G, Chavarrias C, Shemesh N (2018) BOLD-fMRI in the mouse auditory pathway. Neuroimage 165:265-277.

Buhr ED, Yue WW, Ren X, Jiang Z, Liao H-WR, Mei X, Vemaraju S, Nguyen M-T, Reed RR, Lang RA, Yau K-W, Van Gelder RN (2015) Neuropsin (OPN5)-mediated photoentrainment of local circadian oscillators in mammalian retina and cornea. Proc Natl Acad Sci USA 112:13093-13098.

Bureau I, Saint Paul von F, Svoboda K (2006) Interdigitated paralemniscal and lemniscal pathways in the mouse barrel cortex. PLoS Biol 4:e382-11.

Buscone S, Mardaryev AN, Raafs B, Bikker JW, Sticht C, Gretz N, Farjo N, Uzunbajakava NE, Botchkareva NV (2017) A new path in defining light parameters for hair growth: discovery and modulation of photorecep tors in human hair follicle. Lasers Surg Med 45:487-414.

Cassimeris L, Spittle C (2001) Regulation of microtubule-associated proteins. Int Rev Cytol 210:163-226.

Castellano-Pellicena I, Uzunbajakava NE, Mignon C, Raafs B, Botchkarev VA, Thornton MJ (2019) Does blue light restore human epidermal barrier function via activation of Opsin during cutaneous wound healing? Lasers Surg Med 51:370-382.

Chai H, Diaz-Castro B, Shigetomi E, Monte E, Octeau JC, Yu X, Cohn W, Rajendran PS, Vondriska TM, Whitelegge JP, Coppola G, Khakh BS (2017) Neural circuit-specialized astrocytes: transcriptomic, proteomic, morphological, and functional evidence. Neuron 95:531-549.e9.

Chen D, Gibson ES, Kennedy MJ (2013) A light-triggered protein secretion system. J Cell Biol 201:631-640.

Dutra SGV, Paterson A, Monteiro LRN, Greenwood MP, Greenwood M, Amaral LS, Melo MR, Colombari DSA, Colombari E, Reis LC, Hindmarch CCT, Elias LLK, Antunes-Rodrigues J, Murphy D, Mecawi AS (2020) Physiological and transcriptomic changes in the hypothalamic-neurohypophysial system after 24 hours of furosemide-induced sodium depletion. Neuroendocrinology. Advance online publication. doi:10.1159/000505997.

El Massri N, Cullen KM, Stefani S, Moro C, Torres N, Benabid A-L, Mitrofanis J (2018) Evidence for encephalopsin immunoreactivity in interneurones and striosomes of the monkey striatum. Exp Brain Res 236:955-961.

Freund TF, Buzsáki G (1998) Interneurons of the hippocampus. Hippocampus 6:347-470.

Gong S, Zheng C, Doughty ML, Losos K, Didkovsky N, Schambra UB, Nowak NJ, Joyner A, Leblanc G, Hatten ME, Heintz N (2003) A gene expression atlas of the central nervous system based on bacterial artificial chromosomes. Nature 425:917-925.

Guillery RW, Murray Sherman S (2002) Thalamic relay functions and review their role in corticocortical communication: generalizations from the visual system. Neuron 33:163-175.

Halford S, Freedman MS, Bellingham J, Inglis SL, Poopalasundaram S, Soni BG, Foster RG, Hunt DM (2001) Characterization of a novel human opsin gene with wide tissue expression and identification of embedded and flanking genes on chromosome $1 \mathrm{q} 43$. Genomics 72:203-208.

Hartwig HG, van Veen T (1979) Spectral characteristics of visible radiation penetrating into the brain and stimulating extraretinal photoreceptors. J Comp Physiol A Neuroethol Sens Neural Behav Physiol 130:277-282.

Jiao J, Hong S, Zhang J, Ma L, Sun Y, Zhang D, Shen B, Zhu C (2012) Opsin3 sensitizes hepatocellular carcinoma cells to 5- 
fluorouracil treatment by regulating the apoptotic pathway. Cancer Lett 320:96-103.

Jinno S (2011) Regional and laminar differences in antigen profiles and spatial distributions of astrocytes in the mouse hippocampus, with reference to aging. Neuroscience 180:41-52.

Kanaani J, Kolibachuk J, Martinez H, Baekkeskov S (2010) Two distinct mechanisms target GAD67 to vesicular pathways and presynaptic clusters. J Cell Biol 190:911-925.

Kato M, Sugiyama T, Sakai K, Yamashita T, Fujita H, Sato K, Tomonari S, Shichida Y, Ohuchi H (2016) Two opsin 3-related proteins in the chicken retina and brain: a TMT-type opsin 3 is a bluelight sensor in retinal horizontal cells, hypothalamus, and cerebellum. PLoS One 11:1-23.

Kaufman DL, Houser CR, Tobin AJ (1991) Two forms of the gamma -aminobutyric acid synthetic enzyme glutamate decarboxylase have distinct intraneuronal distributions and cofactor interactions. J Neurochem 56:720-723.

Kobat D, Horton NG, Xu C (2011) In vivo two-photon microscopy to 1.6-mm depth in mouse cortex. J Biomed Opt 16:106014.

Koyanagi M, Takada E, Nagata T, Tsukamoto H, Terakita A (2013) Homologs of vertebrate Opn3 potentially serve as a light sensor in nonphotoreceptive tissue. Proc Natl Acad Sci USA 110:4998- 5003.

Lein ES, Hawrylycz MJ, Ao N, Ayres M, Bensinger A, Bernard A, Boe AF, Boguski MS, Brockway KS, Byrnes EJ, Chen L, Chen L, Chen TM, Chin MC, Chong J, Crook BE, Czaplinska A, Dang CN, Datta $\mathrm{S}$, Dee NR, et al. (2007) Genome-wide atlas of gene expression in the adult mouse brain. Nature 445:168-176.

Matyash V, Kettenmann H (2010) Heterogeneity in astrocyte morphology and physiology. Brain Res Rev 63:2-10.

Messing A, Brenner M (2003) GFAP: functional implications gleaned from studies of genetically engineered mice. Glia 43:87-90.

Miller SJ (2018) Astrocyte heterogeneity in the adult central nervous system. Front Cell Neurosci 12:401- 401.

Nayak G, Zhang KX, Vemaraju S, Odaka Y, Buhr ED, Holt-Jones A, Kernodle S, Smith AN, Upton BA, D'Souza S, Zhan JJ, Diaz N, Nguyen MT, Mukherjee R, Gordon SA, Wu G, Schmidt R, Mei X, Petts NT, Batie M, Rao S, et al. (2020) Adaptive thermogenesis in mice is enhanced by opsin 3-dependent adipocyte light sensing. Cell Rep 30:672-686.e8.

Nissilä J, Mänttäri S, Särkioja T, Tuominen H, Takala T, Timonen M, Saarela S (2012) Encephalopsin (OPN3) protein abundance in the adult mouse brain. J Comp Physiol A Neuroethol Sens Neural Behav Physiol 198:833-839.

Oh SW, Harris JA, Ng L, Winslow B, Cain N, Mihalas S, Wang Q, Lau C, Kuan L, Henry AM, Mortrud MT, Ouellette B, Nguyen TN, Sorensen SA, Slaughterbeck CR, Wakeman W, Li Y, Feng D, Ho A, Nicholas E, et al. (2014) A mesoscale connectome of the mouse brain. Nature 508:207-214.

Ohno S, Kuramoto E, Furuta T, Hioki H, Tanaka YR, Fujiyama F, Sonomura T, Uemura M, Sugiyama K, Kaneko T (2012) A morphological analysis of thalamocortical axon fibers of rat posterior thalamic nuclei: a single neuron tracing study with viral vectors. Cereb Cortex 22:2840-2857.

Olinski LE, Lin EM, Oancea E (2020) Illuminating insights into opsin 3 function in the skin. Adv Biol Regul 75:100668-100613.

Ozdeslik RN, Olinski L, Trieu ML, Oprian DD, Oancea E (2019) The human non-visual opsin OPN3 regulates pigmentation of epidermal melanocytes through interaction with MC1R. Proc Natl Acad Sci USA 116:11508-11517.

Paxinos G, Franklin BK (2013) The mouse brain in stereotaxic coordinates. London: Academic Press.

Petryszyn S, Parent A, Parent M (2018) The calretinin interneurons of the striatum: comparisons between rodents and primates under normal and pathological conditions. J Neural Transm (Vienna) 125:279-290.

Pierret T, Lavallée P, Deschênes M (2000) Parallel streams for the relay of vibrissal information through thalamic barreloids. $J$ Neurosci 20:7455-7462.

Pinal CS, Tobin AJ (1998) Uniqueness and redundancy in GABA production. Perspect Dev Neurobiol 5:109-118.

Ransom J, Morgan PJ, McCaffery PJ, Stoney PN (2014) The rhythm of retinoids in the brain. J Neurochem 129:366-376.

Regazzetti C, Sormani L, Debayle D, Bernerd F, Tulic MK, De Donatis GM, Chignon -Sicard B, Rocchi S, Passeron T (2018) Melanocytes sense blue light and regulate pigmentation through opsin-3. J Invest Dermatol 138:171-178.

Sato M, Tsuji T, Yang K, Ren X, Dreyfuss JM, Huang TL, Wang C-H, Shamsi F, Leiria LO, Lynes MD, Yau K-W, Tseng Y-H (2020) Cellautonomous light sensitivity via Opsin3 regulates fuel utilization in brown adipocytes. PLoS Biol 18:e3000630-27.

Shearer KD, Goodman TH, Ross AW, Reilly L, Morgan PJ, McCaffery PJ (2010) Photoperiodic regulation of retinoic acid signaling in the hypothalamus. J Neurochem 112:246-257.

Siljee JE, Wang Y, Bernard AA, Ersoy BA, Zhang S, Marley A, Zastrow Von M, Reiter JF, Vaisse C (2018) Subcellular localization of MC4R with $A D C Y 3$ at neuronal primary cilia underlies a common pathway for genetic predisposition to obesity. Nat Genet 50:180-189.

Sugihara T, Nagata T, Mason B, Koyanagi M, Terakita A (2016) Absorption characteristics of vertebrate non-visual opsin, Opn3. PLoS One 11:e0161215.

Szentagothai J (1963) The structure of the synapse in the lateral geniculate body. Acta Anat 55:166-185.

Tabata M (1982) Persistence of pineal photosensory function in blind cave fish, Astyanax mexicanus. Comp Biochem Physiol A Physiol 73:125-127.

Tamamaki N, Yanagawa Y, Tomioka R, Miyazaki J-I, Obata K, Kaneko T (2003) Green fluorescent protein expression and colocalization with calretinin, parvalbumin, and somatostatin in the GAD67 -GFP knock-in mouse. J Comp Neurol 467:60-79.

Terakita A (2005) The opsins. Genome Biol 6:213.

Van Brunt EE, Shepherd MD, Wall JR, Ganong WF, Clegg MT (1964) Penetration of light into the brain of mammals. Ann NY Acad Sci 117:217-224.

Van Veen T, Hartwig HG, Müller K (1976) Light-dependent motor activity and photonegative behavior in the eel (Anguilla anguilla L.). J Comp Physiol A Neuroethol Sens Neural Behav Physiol 111:209-219.

Viggiani E, Ciesla M, Russo OL (1970) The shielding power of the rat skull to visible light. Experientia 26:850-851.

White JH, Chiano M, Wigglesworth M, Geske R, Riley J, White N, Hall S, Zhu G, Maurio F, Savage T, Anderson W, Cordy J, Ducceschi M, GAIN investigators; Vestbo J, Pillai SG (2008) Identification of a novel asthma susceptibility gene on chromosome 1qter and its functional evaluation. Hum Mol Genet 17:1890-1903.

Wimmer VC, Bruno RM, de Kock CPJ, Kuner T, Sakmann B (2010) Dimensions of a projection column and architecture of VPM and POm axons in rat vibrissal cortex. Cereb Cortex 20:2265-2276.

Yim PD, Gallos G, Perez-Zoghbi JF, Zhang Y, Xu D, Wu A, Berkowitz DE, Emala CW (2019) Airway smooth muscle photorelaxation via opsin receptor activation. Am J Physiol Lung Cell Mol Physiol 316:L82- L93.

Yoshimoto T, Morine Y, Takasu C, Feng R, Ikemoto T, Yoshikawa K, Iwahashi S, Saito Y, Kas hihara H, Akutagawa M, Emoto T, Kinouchi Y, Shimada M (2018) Blue light-emitting diodes induce autophagy in colon cancer cells by opsin 3. Ann Gastroenterol Surg 2:154-161. 